\title{
ON THE MATHEMATICAL WORK OF M. SH. BIRMAN
}

\author{
M. Z. SOLOMYAK, T. A. SUSLINA, AND D. R. YAFAEV
}

\section{Contents}

Brief biography of M. Sh. Birman

$\S 1$. Early papers

$\S 2$. Extension theory for positive definite symmetric operators. Elliptic boundary value problems

$\S 3$. Mathematical scattering theory: the trace class approach

$\S 4$. Spectral shift function

$\S 5$. Double operator integrals

$\S 6$. Function spaces and piecewise-polynomial approximation. Entropy

$\S 7$. Estimates and asymptotics of the spectrum for integral and differential

$\S 8$. Maxwell operator in domains with nonsmooth boundary: asymptotics of the

$\S 9$. Estimates and asymptotics for the number of negative eigenvalues of the

Schrödinger operator

$\S 10$. Discrete spectrum of a perturbed operator in the spectral gaps of the unperturbed one

$\S 11$. Absolute continuity of the spectrum of periodic operators of mathematical

$\S 12$. Threshold properties and homogenization problems for periodic differential operators

$\S 13$. Concluding remarks

List of publications of M. Sh. Birman

1. Papers

2. Books

3. Editorial Activity

List of students of M. Sh. Birman

\section{Brief BIOGRAPhy of M. Sh. Birman}

On July 2, 2009, Mikhail Shlemovich Birman, an outstanding scientist, one of the world leaders in the spectral theory of operators, passed away. The sphere of his scientific interests was wide and diverse. He made great contributions to general theory of 
operators in a Hilbert space, spectral theory of differential operators, mathematical scattering theory, function theory, theory of partial differential equations and other areas. We propose a survey of the most important scientific achievements of M. Sh. Birman.

Mikhail Shlemovich (Solomonovich) Birman was born on January 17, 1928, in Leningrad. His father was a scientist, a specialist in theoretical mechanics, he had a professor position in one of Leningrad technical institutes. Birman's mother was a school teacher.

During World War II the family settled in Sverdlovsk, where Mikhail finished high school. After the war the family returned to Leningrad, and he entered Leningrad Electrical Technical Institute. His professor of mathematics at the Institute was impressed by the outstanding mathematical abilities of the young student and advised him to switch to the Department of Mathematics and Mechanics of the Leningrad State University. Mikhail Shlemovich followed this advice.

During his study at the Department of Mathematics and Mechanics, he specialized in computational methods. The teachers of M. Sh. were Mark Konstantinovich Gavurin, his scientific supervisor, and Leonid Vital'evich Kantorovich. Being still an undergraduate student, M. Sh. took a part time job at the Leningrad Division of Steklov Mathematical Institute, in the laboratory of Kantorovich. Kantorovich distinguished the young colleague for his strong intellect and independent thought and began to give M. Sh. assignements going far beyond standard technical calculations. All this was very important for the mathematical development of M. Sh.. M. Sh. graduated from the Department of Mathematics and Mechanics of the Leningrad State University in 1950. Though he was among the best students, antisemitic trends in the Soviet policy of those days made it impossible for him to become a Ph.D. student.

In 1947 Mikhail Shlemovich married the same-year student Tat'yana Petrovna Il'ina. In 1948 their son Zhenya was born. The happy marriage with Tat'yana Petrovna lasted all his life. She died two years before him. It was due to her love, selflessness, patience, and care that he could fully devote himself to his favourite activity, the study of mathematics.

After graduating from the University M. Sh. worked as an assistant professor at the Department of Mathematics of the Leningrad Mining Institute. In spite of very big amount of pedagogical work, he was actively doing scientific research. In 1954 he successfully defended his Ph.D. Thesis.

Mikhail Shlemovich became one of the most active participants of the Leningrad seminar in mathematical physics organized in the early 1950s by Vladimir Ivanovich Smirnov. This was important for his scientific formation. Practically all his results were first reported at this seminar. Later during many years M. Sh. together with Olga Aleksandrovna Ladyzhenskaya headed this seminar, which is now named after V. I. Smirnov.

In 1956, during "Khrushchev's thaw", on the initiative of Vladimir Ivanovich and Olga Aleksandrovna, Mikhail Shlemovich joined the Division of Mathematical Physics of the Physical Department of Leningrad State University. In 1962 he defended his Doctor of Science Thesis "The spectrum of singular boundary-value problems". At the Division of Mathematical Physics M. Sh. worked for more than 50 years, until the end of his life.

M. Sh. was a brilliant lecturer. He was giving special courses on functional analysis, spectral theory of operators, mathematical scattering theory, and other areas for students specializing in mathematical and theoretical physics. He created an original course of linear algebra for students of the Physical Department. His lectures were not only substantial and well thought out, but also inspiring. M. Sh. was one of the leaders of the Division of Mathematical Physics. He enjoyed the highest prestige. His professional standards, both with regard to the level of results and to the quality of their presentation, were always very high. And at the first place, he applied these standards to himself. 
M. Sh. was always quite attentive to other people. Many his colleagues felt the influence of this highly professional and wise man.

Together with his "brother-in-arms" Mikhail Zakharovich Solomyak, Mikhail Shlemovich created a strong school in the spectral theory of operators, which is internationally known. Under his supervision, more than 20 students defended their Ph.D. Theses, 7 of them later became Doctors of Science. Many of his students became well-known researchers and now work at leading universities in Russia and abroad.

Mikhail Shlemovich Birman was the author of more than 160 research papers and two monographs. He was also active as an editor, a member of the editorial boards of the Russian Academy of Sciences journals "St. Petersburg Mathematical Journal" and "Functional Analysis and its Applications". He edited 13 volumes of "Problems of Mathematical Physics" published by Leningrad State University and several special volumes published by American Mathematical Society.

The results of M. Sh. Birman are well known internationally and are extensively cited by other authors. In 1974 he was invited to give a talk at the International Mathematical Congress (unfortunately, he was not able to accept this invitation because he was not allowed to go abroad). During the last 20 years of his life (when the restictions of the Soviet era for travelling were lifted), he was a plenary speaker at international conferences for many times; he was invited as a visiting scientist to leading world universities and scientific centres.

Despite his infirm health, Mikhail Shlemovich Birman was active in research until the last days of his life. He lived in science and his profession till the end.

The death of Mikhail Shlemovich Birman is irreplaceable loss not only for his relatives, friends, colleagues, students, but for the entire mathematics.

The authors of this paper enjoyed the priviledge of being in close scientific and personal contact with Mikhail Shlemovich. M. Z. Solomyak was his main coathor for almost 40 years. T. A. Suslina and D. R. Yafaev were his students, later the closest collaborators of M. Sh.. We try to reconstruct the picture of wide spectrum of interests and the depth of ideas and results of Mikhail Shlemovich Birman.

Below we give a survey of the most important achivements of M. Sh. Birman. The full list of publications and the list of students of M. Sh. Birman are given in the end of the paper.

\section{$\S 1$. EARLY PAPERS}

The first publications of M. Sh. were related to his work on computational methods at the period when he was a student and worked at the laboratory of L. V. Kantorovich. In the papers [1, 2, 3. inspired by the ideas of Kantorovich, he studied the multistep versions of the successive approximations method and the steepest descent method. We describe here the main idea of the paper [3], where a new method of finding an approximate solution of the equation

$$
A x=\varphi
$$

was suggested and analyzed. Here $A$ is a bounded selfadjoint and positive definite operator in a Hilbert space. Let $m$ and $M$ stand for the lower and upper bounds of the spectrum of $A$. In the traditional approach, equation (1.1) is replaced by the equivalent equation $x=(I-\varepsilon A) x+\varepsilon \varphi$, where $\varepsilon$ is chosen so that $\|I-\varepsilon A\|<1$. The new equation can be solved by the standard successive approximations method. 
In the multistep ( $p$-step) version of the method, equation (1.1) is replaced by the equation $x=B_{p} x+\varphi_{p}$, where

$$
B_{p}=I-\sum_{k=0}^{p-1} \varepsilon_{k}^{(p)} A^{k+1} ; \quad \varphi_{p}=\sum_{k=0}^{p-1} \varepsilon_{k}^{(p)} A^{k} \varphi .
$$

The problem is to make an optimal choice of the coefficients $\varepsilon_{k}^{(p)}$. This choice should minimize the norm of the operator $B_{p}$ and, as a consequence, optimize the rate of convergence of the successive approximations for solution of the new equation.

By developing an idea of his scientific supervisor M. K. Gavurin, M. Sh. suggested to take $B_{p}=T_{p}(A)$, where $T_{p}$ is the Chebyshev polynomial "transferred" to the segment $[m, M]$. M. Sh. gave a detailed analysis of this procedure, showing its advantages compared to the standard approach.

In the papers [1, 2, a similar analysis was given for the multistep analog of the steepest descent method both for solving equation (1.1) and for calculating eigenvalues of the operator $A$.

Many features typical of the scientific style of M. Sh. manifested themselves in these first publications: exhaustive analysis of the problem, extremely clear and transparent exposition, and numerous comments useful for the reader oriented, in the first place, to applications.

\section{§2. EXTENSION THEORY FOR POSITIVE DEFINITE SYMMETRIC OPERATORS. ELLIPTIC BOUNDARY VALUE PROBLEMS}

2.1. In 1952-1954, M. Sh. Birman started his work on the variational theory of elliptic boundary-value problems. He had intensive discussions with S. G. Mikhlin on this subject. At that time, Mikhlin was the most authoritative specialist in this area. Partially influenced by these discussions, M. Sh. undertook an analysis of Trefftz's method, which was suggested in 1926 for the Dirichlet problem in a bounded domain $\Omega \subset \mathbb{R}^{d}$ :

$$
-\Delta u=f \text { in } \Omega, \quad u=0 \text { on } \partial \Omega \text {. }
$$

The method consists in minimizing the Dirichlet integral $\int_{\Omega}|\nabla u|^{2} d x$ among all solutions $u \in H^{1}(\Omega)$ of the equation $-\Delta u=f$ subject to no boundary conditions. The convergence of this method was proved by S. G. Mikhlin in 1950. Together with the standard variational approach, based upon minimizing the functional

$$
\int_{\Omega}\left(|\nabla u|^{2}-2 \operatorname{Re}(\bar{u} f)\right) d x
$$

the Trefftz method enables one to obtain two-sided estimates for the value of the Dirichlet integral of the solution $u$.

M. Sh. attempted to extend the Trefftz method to the case of other boundary-value problems. The main difficulty was to construct a quadratic functional ("Trefftz's functional") that attains its minimum value on the solution of a given boundary value problem.

Elaborating on the question, M. Sh. realized that the general extension theory for symmetric operators plays a central role in such settings. He intensively studied the papers by M. G. Kreı̆n and M. I. Vishik on the subject. He was very much impressed, especially, by the papers of Kreı̆n. In his own words, these papers opened a new world for him. Since then M. Sh. always regarded himself as a "distant student" of M. G. Kreı̆n.

The basic results of M. Sh. in extension theory were presented in the paper [10] (see also a short exposition in [4]). Let $A_{0}$ be a symmetric positive definite operator in a Hilbert space. Denote by $A_{F}$ the Friedrichs extension of $A_{0}$. Let $A$ be an arbitrary positive 
definite extension of $A_{0}$. Developing the ideas of Kreŭn and Vishik, M. Sh. considered the so-called parametric representation of $A$. The role of a parameter was played by a selfadjoint operator $T$ acting in a subspace of the initial space. Typically, this subspace is the kernel Ker $A_{0}^{*}$ of the operator $A_{0}^{*}$ adjoint to $A_{0}$, herewith,

$$
T=\left.\left(A^{-1}-A_{F}^{-1}\right)\right|_{\text {Ker } A_{0}^{*}} \text {. }
$$

M. Sh. gave a detailed analysis of relationships between the spectral properties of $A$ and those of the corresponding "operator parameter" $T$. In [5], he gave an analytic description of the main objects of extension theory as applied to elliptic boundary-value problems. Next, in [6, 8, 11], the general results were applied to construction of the Trefftz functionals for the main boundary value problems for the Laplace and biharmonic operators.

2.2. Soon after that, M. Sh. found another application of his results on extension theory. By the classical Weyl theorem, the compactness of the operator $A_{1}^{-1}-A_{2}^{-1}$ implies that the essential spectra of selfadjoint operators $A_{1}$ and $A_{2}$ coincide. Therefore, the parametric representation (2.2) opens a convenient way to study the stability of the essential spectra of elliptic operators in unbounded domains (the singular elliptic operators).

Main results on this subject were presented in [22. Here we describe the ideas of this paper for the case of the Laplacian in an unbounded domain $\Omega \subset \mathbb{R}^{d}$ with a compact boundary. In this case $A_{0}$ is the Laplace operator defined on the class $\stackrel{\circ}{H}^{2}(\Omega)$ (i.e., on functions in the Sobolev space $H^{2}(\Omega)$ that can be approximated in the $H^{2}(\Omega)$-norm by smooth functions with compact support in $\Omega$ ). The operator $A_{0}^{*}$ is the Laplacian defined on the entire space $H^{2}(\Omega)$, and the subspace Ker $A_{0}^{*}$ is the set of all harmonic functions of class $H^{2}(\Omega)$.

M. Sh. considered also the class $G^{1}(\Omega)$ of all harmonic functions in $H^{1}(\Omega)$. Passing to the quadratic forms and using the parametric representation $(2.2)$, he reduced the stability problem for the essential spectrum to the study of the embedding operator of $G^{1}(\Omega)$ in $L_{2}(\Omega)$. Not only did he prove the required compactness, but also obtained sharp-order estimates for $s$-numbers of the embedding operator. Herewith, M. Sh. introduced the spaces of compact operators with a power-like decay of $s$-numbers, in terms of which the properties of the embedding operator can be described more accurately than in terms of the Schatten-von Neumann classes. Later these classes were called "weak Schatten-von Neumann classes". They are systematically used in general operator theory, as well as in applications.

As a result, M. Sh. proved the stability of the essential spectrum under perturbation of a compact part of the boundary or a perturbation of the boundary condition on such a part of the boundary. Using the Kato-Rosenblum theorem (see Subsection 3.1 below), he obtained a similar result for the absolutely continuous part of the spectrum. This required overcoming additional technical difficulties.

M. Sh. always treated the paper 22 as one of his main achievements in spectral theory of differential operators. That is why he was distressed about the absence of the English translation of that paper (it was translated only in 2008, in connection with the 80th birthday of M. Sh.). Much later, in the 1990s, during his first visit to New York, he was pleased to know that this paper was "nonofficially" translated into English in the 1960s in the Courant Institute. That "nonofficial" version was widely used both in USA and in Europe.

2.3. One of the deepest and most famous papers of M. Sh. is [19] (a short preliminary version appeared in [15]). In that paper, he studied the stability of the essential spectrum 
with respect to changing the coefficients of the operator. That paper was written and published before the paper 22 described above. Its main content is the investigation of the spectrum of the Schrödinger operator $-\Delta+V$ in $L_{2}\left(\mathbb{R}^{d}\right)$ in dependence on the behavior of the potential. This investigation was prefaced by preliminary material, where some basic problems of general theory of semibounded selfadjoint operators were set up and solved. In particular, the notions of relative boundedness and relative compactness for an operator "in the sense of quadratic forms" were systematically used there (though the terms themselves were not introduced).

Here we list main results of the paper [19].

1. The essential spectrum of a selfadjoint operator $A \geq 0$ is stable with respect to relatively compact perturbations (in the sense of forms). Let $V \geq 0$ be such a perturbation. Then the negative spectrum of the operator $A-V$ is discrete. For $\gamma>0$ we consider an "energy space" $\mathcal{H}_{\gamma}(A)$, which is the completion of the domain of $A$ with respect to the norm $\left\|(A+\gamma I)^{1 / 2} x\right\|$. Suppose that the operator $T_{\gamma}(A, V)$ generated by the form $(V x, x)$ in the space $\mathcal{H}_{\gamma}(A)$ is compact. M. Sh. discovered that the number of eigenvalues of the operator $A-V$ lying to the left of the point $-\gamma$ is equal to the number of eigenvalues of the operator $T_{\gamma}(A, V)$ lying to the right of the point 1 .

Two years later this identity was rediscovered by Schwinger; this is the famous Birman-Schwinger principle. Until now, this principle remains the starting point for the study of the eigenvalues of operators with nonempty essential spectrum. Most frequently, this principle is used in quantum mechanics problems. The operator $T_{\gamma}(A, V)$ defined above is usually called the "Birman-Schwinger operator" corresponding to the spectral problem under investigation.

2. The Birman-Schwinger principle opens way to quantitative investigation of the discrete spectrum of singular differential operators. As an application of his general results, M. Sh. proved the famous estimate for the number of negative eigenvalues of the Schrödinger operator $-\Delta-V$ in $L_{2}\left(\mathbb{R}^{3}\right)$ :

$$
N_{-}(-\Delta-V) \leq \frac{1}{16 \pi^{2}} \int_{\mathbb{R}^{3}} \int_{\mathbb{R}^{3}} \frac{V_{+}(x) V_{+}(y)}{|x-y|^{2}} d x d y .
$$

Here $2 V_{+}(x)=V(x)+|V(x)|$. This was the first quantitative estimate for the negative spectrum of the Schrödinger operator in the multidimensional case. It was independently found by Schwinger and is usually called the "BirmanSchwinger estimate".

3. The Birman-Schwinger principle remains true also for the number of all negative eigenvalues of the operator $A-V$ if $A>0$ and the point zero is the bottom of the essential spectrum of $A$. However, in this situation two cases should be distinguished: that in which the form $(A x, x)$ is closable in the main Hilbert space, and that in which it is not. In the second case, $A$ is said to have a zeroenergy resonance. Herewith, the definition of the Birman-Schwinger operator $T_{0}(A, V)$ becomes somewhat more complicated. Moreover, in the identity for the number of negative eigenvalues of the operator $A-V$ (which expresses the Birman-Schwinger principle), one should sum up the number of the eigenvalues of $T_{0}(A, V)$ greater than 1 and the multiplicity of the zero-energy resonance. In particular, it follows that if $A$ has a virtual level, then the negative spectrum of the operator $A-V$ is not empty for arbitrary negative perturbation $-V$.

4. M. Sh. found very general conditions for the discreteness or finiteness of the negative spectrum of the operator family $A-\alpha V$ for all values of the parameter $\alpha>0$ (called the coupling constant). In many important cases, these conditions 
turned out to be necessary and sufficient for the corresponding behavior of the negative spectrum.

In order to apply the general results of [19] to the operators $H_{l, \alpha V}=(-\Delta)^{l}-\alpha V$, a question from the theory of function spaces had to be settled. Specifically, one needed to describe the weight functions $V$ for which the Sobolev space $H^{l}\left(\mathbb{R}^{d}\right)$, or the "homogeneous" Sobolev space $\mathcal{H}^{l}\left(\mathbb{R}^{d}\right)$, is compactly embedded into the weighted space $L_{2, V}\left(\mathbb{R}^{d}\right)$. The first embedding corresponds to the discreteness and the second to the finiteness of the negative spectra of the operators $H_{l, \alpha V}$ (for all $\alpha>0$ at once).

M. Sh. studied these questions in the same papers [15, 19], and also in the paper [18] written jointly with B. S. Pavlov. For the case where $2 l>d$, a complete description of admissible weights was found. For $l=1, d \geq 2$ (i.e., for the case of a multidimensional Schrödinger operator) some necessary and (separate) sufficient conditions of compactness were obtained for such embeddings. Later, a necessary and sufficient condition of the embedding compactness (for the case of $l=1$ ) was found by V. G. Maz'ya; this condition involves capacity.

2.4. Describing this period of M. Sh. Birman's scientific activity, it is impossible to bypass the paper 25] (joint with his student G. E. Skvortsov) on the index of the Dirichlet Laplacian in plain domains with edges.

In the early 1960s, the theory of elliptic boundary value problems in bounded domains with smooth boundary was well developed, and M. Sh. widely applied its results in his paper 22. However, he always used a variational definition of an operator, which does not require any smoothness of the boundary (in the case of the Dirichlet problem).

A natural question was: do the results of "smooth" theory remain true for the case of "bad" boundaries? At that time, examples were known showing that, in a plane region $\Omega$ with a piecewise smooth boundary the Dirichlet Laplacian defined on the natural domain $H^{2}(\Omega) \cap \stackrel{\circ}{H}^{1}(\Omega)$ may fail to be selfadjoint. However, no general results had been known before the paper [25, where it was shown that the index of the Dirichlet Laplacian is equal to the number of internal edges greater than $\pi$. This paper stimulated investigations of elliptic boundary value problems in domains with nonsmooth boundary. Soon the corresponding theory was created in the papers of V. A. Kondrat'ev, V. G. Maz'ya, B. A. Plamenevskil and other mathematicians.

\section{§3. Mathematical SCATtering theory: the trace Class APPROACH}

3.1. The trace class approach in scattering theory originated in the papers by T. Kato and M. Rosenblum (1957), where the following fundamental result was proved. Let $A$ and $B$ be selfadjoint operators in a Hilbert space $\mathcal{H}$, and let $P_{\mathrm{ac}}(A)$ and $P_{\mathrm{ac}}(B)$ be the orthogonal projections onto the absolutely continuous subspaces $\mathcal{H}_{\mathrm{ac}}(A)$ and $\mathcal{H}_{\mathrm{ac}}(B)$ of $A$ and $B$, respectively. Assume that the difference $B-A$ belongs to the trace class $\mathfrak{S}_{1}$. Then the strong limits

$$
\underset{t \rightarrow \pm \infty}{\mathrm{s}-\lim _{\mathrm{D}}} \exp (i B t) \exp (-i A t) P_{\mathrm{ac}}(A)=: W_{ \pm}(B, A)
$$

exist. They are called the wave operators. The operators $W_{ \pm}(B, A)$ are automatically isometric on $\mathcal{H}_{\mathrm{ac}}(A)$, and $B W_{ \pm}(B, A)=W_{ \pm}(B, A) A$. Since the assumptions of the Kato-Rosenblum theorem are symmetric, the wave operators $W_{ \pm}(A, B)$ also exist and hence the range of operators $W_{ \pm}(B, A)$ coincides with the subspace $\mathcal{H}_{\mathrm{ac}}(B)$ (in this case the wave operators are said to be complete). Thus, under the condition $B-A \in \mathfrak{S}_{1}$, the absolutely continuous parts of the operators $A$ and $B$ are unitarily equivalent. 
Note that it is not difficult to show the existence of the weak wave operators

$$
\underset{t \rightarrow \pm \infty}{\mathrm{w}-\lim _{\mathrm{ac}}} P_{\mathrm{ac}}(B) \exp (i B t) \exp (-i A t) P_{\mathrm{ac}}(A) \text {. }
$$

It suffices to apply the following estimate (Rosenblum's lemma):

$$
\int_{-\infty}^{\infty}\left\|G e^{-i A t} f\right\|^{2} d t \leq C(f)\|G\|_{\mathfrak{S}_{2}}^{2}
$$

which is true for all operators $G$ of the Hilbert-Schmidt class $\mathfrak{S}_{2}$ on some set of elements $f$ dense in $\mathcal{H}_{\text {ac }}(A)$ (this set does not depend on $G \in \mathfrak{S}_{2}$ ). However, it is a substantial problem to prove that the weak wave operators are isometric (which ensures the existence of the strong limits (3.1)).

If the limits (3.1) do exist, then the scattering operator

$$
\mathbf{S}:=W_{+}^{*}(B, A) W_{-}(B, A)
$$

commutes with $A$ and thus acts as multiplication by an operator-valued function $S(\lambda)$ in the representation of $\mathcal{H}_{\mathrm{ac}}(A)$ diagonal for $A$. The scattering operator $\mathbf{S}$ and the scattering matrix $S(\lambda)$ first appeared in the theory of scattering of quantum particles, which explains why the perturbation theory of the absolutely continuous spectrum is called "scattering theory".

The simplest quantum system is described by the Schrödinger operator

$$
B=-\Delta+V(x)
$$

acting in the space $\mathcal{H}=L_{2}\left(\mathbb{R}^{d}\right)$, where $V(x)$ is a real-valued function (the potential energy) that decays sufficiently rapidly at infinity. Thus, $B$ is a perturbation of the kinetic energy operator $A=-\Delta$ by a multiplication operator which is never compact. Therefore, the Kato-Rosenblum theorem cannot be applied to this important case directly. When the papers of T. Kato and M. Rosenblum appeared, the problem of application of their theorem to differential operators was immediately posed. This problem was studied by T. Kato himself, by S. T. Kuroda, and by many other mathematicians. The contribution of M. Sh. Birman to this highly competitive area was crucial.

3.2. For M. Sh., the study of the absolutely continuous spectrum $\sigma_{\text {ac }}$ was a natural continuation of his work on the essential spectrum. The connecting link was the paper [22] where the invariance of $\sigma_{\mathrm{ac}}$ was verified for perturbations of the boundary or of the boundary conditions for elliptic operators in unbounded domains (see the discussion in Subsection 2.2).

The initial and, as it turned out later, very fruitful idea of M. Sh. was to consider suitable functions $\varphi$ of operators $A$ and $B$ (for example, the operators $A^{-1}$ and $B^{-1}$, when $\varphi(\lambda)=\lambda^{-1}$ ) and to apply the Kato-Rosenblum theorem to the pair of operators $\varphi(A), \varphi(B)$. The invariance of the absolutely continuous spectrum allowed M. Sh. to conjecture that, under the assumption

$$
\varphi(B)-\varphi(A) \in \mathfrak{S}_{1}
$$

not only do we have $\sigma_{\mathrm{ac}}(B)=\sigma_{\mathrm{ac}}(A)$, but also the wave operators $W_{ \pm}(B, A)$ exist and

$$
W_{ \pm}(\varphi(B), \varphi(A))=W_{ \pm}(B, A)
$$

(or $W_{ \pm}(\varphi(B), \varphi(A))=W_{\mp}(B, A)$, depending on the sign of $\varphi^{\prime}(\lambda)$ ). This result, proved by M. Sh. Birman in [23, 27] for a wide class of functions $\varphi$, was later called the invariance principle.

At the same period, in the joint paper 24] of M. G. Krĕn and M. Sh. Birman, the Kato-Rosenblum theorem was carried over to unitary operators. This corresponds to the invariance principle for the fractional-linear function $\varphi$, when $\varphi(A)$ and $\varphi(B)$ are the 
Cayley transforms of the operators $A$ and $B$. The Birman-Krel̆ theorem implies that, for a pair of selfadjoint operators $A, B$, the wave operators $W_{ \pm}(B, A)$ exist if the difference of their resolvents belongs to the trace class. This is an important generalization of the Kato-Rosenblum theorem, which can be applied directly to the Schrödinger operator.

3.3. In addition to the formulation in terms of (nonstationary) wave operators (3.1), scattering theory admits also a stationary formulation, where the unitary groups in the definition (3.1) are replaced by the resolvents of the operators $A$ and $B$. Herewith, instead of the limits as $t \rightarrow \pm \infty$, one has to study the limits of resolvents as the complex spectral parameter approaches the real axis. This method allows one to obtain formulas for the main objects of the theory, which is important for applications to physics. A lot of credit must go to M. Sh. developing the stationary approach consistently in the trace class scattering theory; see the papers [30, 36] written jointly with his student S. B. Entina. From an analytic point of view, the method of these papers leans upon the existence almost everywhere of the limits for the resolvent of any selfadjoint operator sandwiched between Hilbert-Schmidt operators. This result is also important on its own sake. The stationary approach ensures that the weak wave operators are isometric, which implies the existence of the strong limits (3.1).

In 31, 41, M. Sh. introduced an important notion of the local wave operators associated with some interval of the spectral axis, and found conditions for their existence, also of local nature. Using these conditions, he obtained very general conditions of the existence of the "global" wave operators (3.1). It turned out that, besides the condition that $E_{X}(B)(B-A) E_{X}(A) \in \mathfrak{S}_{1}$ for any bounded interval $X$ (where $E_{X}(A)$ and $E_{X}(B)$ are spectral projections of the operators $A$ and $B$ ), it suffices to impose some condition on the domains of $A$ and $B$ or on the domains of certain functions of $A$ and $B$. For instance, it suffices to assume that $\mathcal{D}(A)=\mathcal{D}(B)$ or $\mathcal{D}\left(|A|^{1 / 2}\right)=\mathcal{D}\left(|B|^{1 / 2}\right)$.

For operators $A$ and $B$ acting in different spaces $\mathcal{H}$ and $\mathcal{K}$, a natural generalization of the definition (3.1) is given by

$$
\underset{t \rightarrow \pm \infty}{\operatorname{s}-\lim _{t \rightarrow \infty}} \exp (i B t) J \exp (-i A t) P_{\mathrm{ac}}(A)=: W_{ \pm}(B, A ; J),
$$

where $J: \mathcal{H} \rightarrow \mathcal{K}$ is a bounded operator. This definition was introduced by T. Kato, but the first efficient conditions (of trace class type) for the existence of the operators (3.2) were found by M. Sh. Birman in the paper [42], written jointly with his student A. L. Belopolskiu. In that paper, the techniques of [36] and [41] were developed further. The approach of 42. (see also [47]) made it possible to find conditions that ensure not merely the existence of the wave operators (3.2), but also their isometric property and completeness. Much later D. Pearson found a direct generalization of the Kato-Rosenblum theorem. The Pearson theorem claims that the limits (3.2) exist if the operator $B J-J A$ belongs to the trace class. Its proof is based on a subtle application of the Rosenblum lemma.

Still much later, M. Sh. returned to this topic in the paper 94 (written jointly with D. R. Yafaev), where the stationary approach was developed in a very general framework (without any assumptions of trace class type or smooth type). In [94, the wave operators (3.2) were considered. It turned out that the role of the isometric property for such operators is played by the identity

$$
W_{ \pm}^{*}(B, A ; J) W_{ \pm}(B, A ; J)=W_{ \pm}\left(A, A ; J^{*} J\right),
$$

proved in 94]. In particular, the method of 94] leads to the stationary proof of the Pearson theorem. 
3.4. Thus, M. Sh. Birman developed the abstract trace class scattering theory to the level where it could be directly applied to differential operators (part of this work was done jointly with his students). These applications were summarized in the papers [44, 46, where a wide class of equations of mathematical physics was considered, and also in [52], where the problems of the perturbation of the medium were studied. Moreover, it was allowed that the order of the perturbation $V=B-A$ be greater than the order of the "unperturbed" operator $A$. In particular, in [46], the existence and completeness of the wave operators was proved in the case of the operators $A=-\Delta$ and $V=\Delta v(x) \Delta$ acting in $\mathcal{H}=L_{2}\left(\mathbb{R}^{d}\right)$, under the assumption that $v(x) \geq 0$ and

$$
\int_{\mathbb{R}^{d}}\left(v^{2}(x)+|\nabla v(x)|^{2}+(\Delta v(x))^{2}\right)\left(1+|x|^{2}\right)^{\alpha}<\infty, \quad 2 \alpha>d .
$$

We present a result of $[52]$. In $L_{2}\left(\mathbb{R}^{d} ; \mathbb{C}^{m}\right)$, consider a homogeneous matrix elliptic operator $A_{0}$ with constant degeneration (i.e., its symbol $a(\xi)$ is of constant rank for $\xi \neq 0)$. Assume that the $(m \times m)$-matrices $M_{0}$ and $M(x)$ are positive definite, $M(x)$ is a continuous function of $x, 0<c_{0} \leq M(x) \leq c_{1}<\infty$, and $M(x)-M_{0} \rightarrow 0$ as $|x| \rightarrow \infty$. The operators $A$ and $B$ are defined by the relations $A=M_{0}^{-1} A_{0}$ and $B=M(x)^{-1} A_{0}$ and act in the spaces $\mathcal{H}_{0}$ and $\mathcal{H}$ with the inner products

$$
(f, g)_{0}=\int_{\mathbb{R}^{d}}\left(M_{0} f(x), g(x)\right) d x \quad \text { and } \quad(f, g)=\int_{\mathbb{R}^{d}}(M(x) f(x), g(x)) d x
$$

respectively. Let $I_{0}: \mathcal{H}_{0} \rightarrow \mathcal{H}$ be the identity operator. Then, under the assumption

$$
\int_{\mathbb{R}^{d}}\left|M(x)-M_{0}\right|^{2}\left(1+|x|^{2}\right)^{\alpha} d x<\infty, \quad 2 \alpha>d,
$$

the wave operators $W_{ \pm}\left(B, A ; I_{0}\right)$ exist, are isometric and complete. This result can directly be applied to the Maxwell equations.

The paper [99], which was written much later, is devoted to applications of the trace class method under minimal assumptions about the unperturbed operator. A typical result is the following. Let

$$
A=(-\Delta)^{l}+V_{0}(x), \quad B=(-\Delta)^{l}+V(x),
$$

where $l$ is any natural number, and $V_{0}$ and $V$ are arbitrary bounded real-valued functions such that

$$
V(x)-V_{0}(x)=O\left(|x|^{-d-\varepsilon}\right), \quad|x| \rightarrow \infty, \quad \varepsilon>0 .
$$

Then the wave operators $W_{ \pm}(B, A)$ exist and are complete. We emphasize that, without any special assumptions on the function $V_{0}(x)$, it is impossible to carry out efficient spectral analysis for the unperturbed operator $A$. However, this is not important for the trace class approach. This is a typical advantage compared to the smooth approach, which is based on eigenfunction expansion for the operator $A$.

\section{$\S 4$. Spectral Shift FunCtion}

4.1. The spectral shift function $\xi(\lambda)=\xi(\lambda ; B, A)$ may be introduced by the relation

$$
\operatorname{Tr}(\varphi(B)-\varphi(A))=\int_{-\infty}^{\infty} \varphi^{\prime}(\lambda) \xi(\lambda) d \lambda, \quad \xi(\lambda)=\xi(\lambda ; B, A),
$$

called the trace formula. The concept of the spectral shift function in perturbation theory arose in the beginning of $1950 \mathrm{~s}$ in the physics literature in the papers of I. M. Lifshitz. Its mathematical theory was created shortly after by M. G. Kreŭn, who proved relation (4.1) for a pair of selfadjoint operators $A$ and $B$ with a trace class difference $V=B-A$ 
and a wide class of functions $\varphi$ (including the function $\varphi(\lambda)=\lambda$ ). M. G. Krein proved the inequality

$$
\int_{-\infty}^{\infty}|\xi(\lambda)| d \lambda \leq\|V\|_{\mathfrak{S}_{1}}
$$

for the spectral shift function. The work of M. G. Krein was based on the methods of complex analysis.

4.2. Much later in 1975 in the paper [55], M. Sh. Birman and M. Z. Solomyak tried to find a proof of the trace formula in the spirit of "real analysis". From an analytic point of view, their approach was based on the techniques of double operator integrals (see $\S 5$ below). M. Sh. Birman and M. Z. Solomyak found a new proof of formula (4.1), but with a distribution $\xi$ (the derivative of a measure). It is important that on this way new representations for the spectral shift function in terms of the spectral measure of the operator family $B_{\gamma}=A+\gamma V$ were obtained. Namely, in [55] it was shown that

$$
\int_{\delta} \xi(\lambda) d \lambda=\int_{0}^{1} \operatorname{Tr}\left(E_{\delta}\left(B_{\gamma}\right) V\right) d \gamma
$$

for any interval $\delta=(a, b)$. It follows that

$$
\xi(\lambda)=\frac{d}{d \lambda} \int_{0}^{1} \operatorname{Tr}\left(E_{(-\infty, \lambda)}\left(B_{\gamma}\right) V\right) d \gamma
$$

for almost every $\lambda \in \mathbb{R}$. This formula is convenient for obtaining estimates for the spectral shift function, such as the estimate

$$
\operatorname{Tr}\left(E_{(-\infty, \lambda)}(B) V\right) \leq \int_{-\infty}^{\lambda} \xi(\mu) d \mu \leq \operatorname{Tr}\left(E_{(-\infty, \lambda)}(A) V\right) .
$$

Note that, as $\lambda \rightarrow \infty$, this inequality yields the trace formula (4.1) for the function $\varphi(\lambda)=\lambda$.

4.3. The relationship between the spectral shift function and the scattering matrix $S(\lambda)=S(\lambda ; B, A)$ was discovered in the paper [24] by M. Sh. Birman and M. G. KreĬn. Namely, in 24] it was shown that

$$
\int_{\sigma_{\mathrm{ac}}(A)}\|S(\lambda)-I\|_{\mathfrak{S}_{1}} d \lambda \leq 2 \pi\|V\|_{\mathfrak{S}_{1}}
$$

which implies that

$$
S(\lambda)-I \in \mathfrak{S}_{1}
$$

(so that the determinant of $S(\lambda)$ is well defined) for almost every $\lambda \in \sigma_{\mathrm{ac}}(A)$. A link between $\xi(\lambda)$ and $S(\lambda)$ is given by the identity

$$
\text { Det } S(\lambda)=\exp (-2 \pi i \xi(\lambda)) \text {, }
$$

called the Birman-Krein formula. Often this formula is regarded as a definition of the spectral shift function on the absolutely continuous spectrum.

Relation (4.3) shows that the spectrum of the unitary operator $S(\lambda)$ consists of eigenvalues lying on the unit circle that may accumulate only to the point 1 . This statement can be refined for the sign-definite perturbations. Namely, in [24] it was shown that there is no accumulation from above (from below) if $V \geq 0$ (if $V \leq 0$ ). In the abstract framework, the eigenvalues of $S(\lambda)$ play the role of phase shifts for the Schrödinger operator with a radial potential.

The short note 24] (and the report 28]) laid foundations for further investigation of the spectral shift function $\xi(\lambda)$ and the scattering matrix $S(\lambda)$, both by students of 
M. Sh. Birman and by researchers from quite different mathematical schools. M. Sh. himself also maintained interest in that field for a long time. In the paper 80 joint with D. R. Yafaev (see also the short note [78, and the "heuristic" exposition [81]) the asymptotics of the spectrum of the scattering matrix for the Schrödinger operator with a general (not radial) potential was found. In 121, this result was extended to perturbations of the Schrödinger operator with a periodic potential.

The papers of M. Sh. Birman had extremely serious influence on further development of the scattering theory and spectral shift function theory. The modern state of the art in the theory of the spectral shift function and spectral properties of the scattering matrix was described in the detailed surveys [115, 136, and [116.

\section{$\S 5$. Double operator integrals}

5.1. A double operator integral (DOI) is an expression of the form

$$
Q=\iint \psi(\lambda, \mu) d F(\mu) T d E(\lambda)
$$

where $d E$ and $d F$ are spectral measures in a Hilbert space, $T$ is a bounded operator, and $\psi$ is a scalar function (the "symbol" of DOI). Here integrals are taken over some sets on which the measures $d E, d F$ are defined (we have omitted these sets in the notation). Being defined in an appropriate way, for given $\psi, d E$, and $d F$ the integral (5.1) determines a linear mapping $\Psi: T \mapsto Q$. Such mappings acting between appropriate classes of operators are usually called "transformers". The DOI's were introduced by Yu. L. Daletskiı̌ and S. G. Kreln in 1956 in connection with the differentiation problem for operator-valued functions depending on a real parameter.

The interest of M. Sh. Birman to DOI's was motivated by his work on the stationary approach to scattering theory (in the early 1960s). The development of this approach required to analyze many natural questions, unresolved at that time. In particular, it was necessary to study the following question: for what functions $\varphi$ the inclusion $B-A \in \mathfrak{S}_{1}$ (where $A, B$ are selfadjoint operators) implies that $\varphi(B)-\varphi(A) \in \mathfrak{S}_{1}$ ? In connection with this problem, M. G. KreIn attracted M. Sh.'s attention to the paper of Yu. L. Daletskiı̌ and S. G. Kreı̆n. In M. G.'s opinion, that paper could be useful in this range of questions. M. Sh. attracted M. Z. Solomyak to the study of this subject; it was the beginning of their joint work, which lasted, with small breaks, for almost 40 years.

In the paper of Yu. L. Daletskiı and S. G. Krein, the approach to the integrals (5.1) was based on the representation

$$
Q=\int K(\lambda) d E(\lambda)
$$

where $K(\lambda)=\left(\int \psi(\lambda, \mu) d F(\mu)\right) T$ is an operator-valued function of the parameter $\lambda$. After that, formal integration by parts reduces the problem to integration of the operatorvalued function $K^{\prime}(\lambda) E(\lambda)$ in $\lambda$. This approach leads to rather rough estimates of the integral (5.1), and therefore it was unsuitable for main applications.

5.2. In the approach developed by M. Sh. together with M. Z. Solomyak [33, 37, 59] (see also the survey [152] written much later), the initial objects are DOI's in the HilbertSchmidt class $\mathfrak{S}_{2}$. In the Hilbert space $\mathfrak{S}_{2}$, the operators of multiplication by $F(\cdot)$ from the left and by $E(\cdot)$ from the right commute and generate a spectral measure in $\mathfrak{S}_{2}$. The integral (5.1) for $T \in \mathfrak{S}_{2}$ is defined as the integral of $\psi$ with respect to this spectral measure applied to the element $T$. This simple but crucial argument directly implies that DOI's in $\mathfrak{S}_{2}$ keep all properties of integrals with respect to a spectral measure. In 
particular, a DOI in $\mathfrak{S}_{2}$ is well defined for any bounded symbol, and we have $\|Q\|_{\mathfrak{S}_{2}} \leq$ $\sup |\psi|\|T\|_{\mathfrak{S}_{2}}$.

However, the boundedness of the symbol is not sufficient for the boundedness of the transformer (5.1) in other operator classes, in particular, in the algebra $\mathfrak{B}$ of all bounded operators. In [33, 37, 59], it was shown that the boundedness of the transformer (5.1) in $\mathfrak{B}$ is closely related to estimates of the trace class norm of integral operators with the kernel $\psi(\lambda, \mu)$ acting in weighted $L_{2}$-spaces. Such estimates depending on the properties of a weight function were unknown before. They were obtained in a series of papers described below in $\S 7$.

Also, a rather general "factorizational" condition on the symbol $\psi$ sufficient for the boundedness of the transformer (5.1) in $\mathfrak{B}$ was found. Later, V. V. Peller showed that this condition is also necessary.

By duality, the boundedness of the transformer (5.1) in $\mathfrak{B}$ is equivalent to its boundedness in the trace class $\mathfrak{S}_{1}$. The action of this transformer in other classes, including the Schatten-fon Neumann ideals $\mathfrak{S}_{p}$, was studied by linear interpolation. Thus, a consistent theory of DOI's as transformers acting between various symmetrically normed operator ideals was developed.

5.3. A DOI can be interpreted as a rather general "multiplier transformation". Consider the set of all integral operators acting from $L_{2}(\Lambda, d \lambda)$ to $L_{2}(M, d \mu)$, where $(\Lambda, d \lambda)$ and $(M, d \mu)$ are two spaces with measure. For a given function $\psi(\lambda, \mu)$, we consider the linear transformation that takes an integral operator $T$ with the kernel $T(\lambda, \mu)$ into the integral operator $Q$ with the kernel $\psi(\lambda, \mu) T(\lambda, \mu)$. In the paper [37, it was shown that this transformation can be written as a DOI with the symbol $\psi(\lambda, \mu)$. Moreover, any DOI can be represented as such a multiplier transformation, but with an operator-valued kernel $T(\lambda, \mu)$. The results about DOI's make it possible to estimate the norms of the operator $Q$ in various classes $\mathfrak{S}_{p}$ in terms of the corresponding norms of $T$.

In the cited papers written in 1965-1973, it was shown that many analytic objects of different nature may be interpreted as DOI's with appropriate choice of spectral measures. For instance, if $d E$ is the spectral measure of a multiplication operator and $d F$ is the spectral measure of a differential operator, and $T=I$, then the DOI (5.1) is a pseudodifferential operator with symbol $\psi$. New boundedness conditions for pseudodifferential operators in $L_{2}\left(\mathbb{R}^{d}\right)$ were obtained with the help of DOI's. Another bright example is representation of a Volterra operator in terms of its imaginary component, which is a principal object of study in the theory of Volterra operators. This representation can also be written in terms of DOI's.

5.4. Main applications of DOI's theory are related to the spectral perturbation theory. At the first place, they are based on the following important formula:

$$
\varphi(B)-\varphi(A)=\iint \frac{\varphi(\lambda)-\varphi(\mu)}{\lambda-\mu} d E(\lambda)(B-A) d F(\mu) .
$$

Here $A$ and $B$ are selfadjoint operators, $d E$ and $d F$ are their spectral measures, and $\varphi$ is a function on $\mathbb{R}$. This formula was proved under very general conditions on a function $\varphi$, and moreover, unbounded operators $A$ and $B$ were admitted. It was only assumed that their difference is bounded. In particular, the initial problem (when the operator $\varphi(B)-\varphi(A)$ belongs to the trace class) was solved with the help of (5.2). An analog of formula (5.2) was also obtained for commutators.

Formula (5.2) implies the differentiation formula

$$
\left.\varphi^{\prime}(A+t T)\right|_{t=0}=\iint \frac{\varphi(\lambda)-\varphi(\mu)}{\lambda-\mu} d E(\lambda) T d E(\mu),
$$


which had been proved before in the paper by Yu. L. Daletskiŭ and S. G. Kreŭn under rather restrictive conditions on $\varphi$ and only for bounded operators $A, B$. In the papers 33, 37, 59, these conditions were extended significantly. It was also proved that under the condition $B-A \in \mathfrak{S}_{p}$ there exists a derivative in the $\mathfrak{S}_{p}$-norm.

The sharpest analytic conditions on $\varphi$ that ensure (5.3) have been obtained later by V. V. Peller.

An application of DOI's connected with (5.3) was already mentioned in Subsection 4.2. It is the deduction of the new representation (4.2) for the integrated spectral shift function.

Yet another important application of formula (5.3) was found in the paper 64] by M. Sh. Birman, L. S. Koplienko, and M. Z. Solomyak. Namely, it was shown that for any nonnegative operators $A$ and $B$ and for $\alpha \in(0,1), p \in(0, \infty]$ the estimate

$$
\left\|\left(B^{\alpha}-A^{\alpha}\right)_{ \pm}\right\|_{\mathfrak{S}_{p}} \leq\left\|\left((B-A)_{ \pm}\right)^{\alpha}\right\|_{\mathfrak{S}_{p}}
$$

is true with the coefficient 1 on the right-hand side!

\section{§6. Function spaces And PieceWise-Polynomial APPROXIMATION. ENTROPY}

6.1. The interest of M. Sh. to the theory of function spaces, especially, to the embedding properties of Sobolev spaces, originates from his work on spectral theory of differential operators. His first results in this field were mentioned briefly in $\S 2$. They were considerably extended and refined in the mid-1960s, when M. Sh. together with M. Z. Solomyak started a systematic investigation of quantitative characteristics of such embeddings. This study was based on a new approach to approximation of functions in Sobolev spaces $W_{p}^{l}$, which was developed in the paper [38; ; detailed exposition can be found in the lectures [62. (English translation is the book B3] in the list of books). This method can be regarded as a multidimensional analog of spline approximation with nonfixed nodes.

Below we give a brief description of this method for the case of $l=1$. Let $X$ be a Banach space of functions on the unit cube $\mathbb{Q}^{d} \subset \mathbb{R}^{d}$. Suppose that the Sobolev space $W_{p}^{1}\left(\mathbb{Q}^{d}\right)$ is compactly embedded in $X$. A given function $u \in W_{p}^{1}\left(\mathbb{Q}^{d}\right)$ is approximated in the norm of the space $X$ by a suitable piecewise-constant function $f$. The construction of $f$ is determined by the choice of a partition of $\mathbb{Q}^{d}$ into a finite number of smaller cubes. Their sizes can be arbitrary, only the number of cubes is under control: it should not exceed a given number $n$. We take $f$ equal to the mean value of $u$ on each cube of the partition. Thus, the function $f$ is completely determined by the choice of the partition. The next step is crucial: for a given $n$, we minimize the error $\|u-f\|_{X}$ among all partitions subject to the above restriction. An algorithmic partitioning procedure, suggested in [38], gives an optimal order of approximation for a wide class of metrics $\|\cdot\|_{X}$.

This approach turned out to be quite flexible and efficient. The results obtained by this method are of two types. For results of the first type, the choice of the partition depends on the function to be approximated; this makes the process nonlinear. For results of the second type, approximations in the weighted spaces $L_{q, V}$ are considered, and the choice of the partition depends on the weight function $V$. The corresponding algorithm is linear with respect to the function to be approximated.

The results obtained by the second algorithm have many important applications in the spectral theory of differential and integral operators; they are described below in $\S 7$. 
6.2. In the concluding part of this section, we describe main applications of the nonlinear approximation procedure. They concern estimates of the Kolmogorov $\varepsilon$-entropy $\mathcal{H}(\varepsilon)$ of the embeddings of Sobolev classes in the spaces $C$ or $L_{q}, q<\infty$.

We discuss the problem of embedding in $C$ for the case where $d=1$ and $l=1$. It had been well known by the end of 1950 -s that for the unit ball of $C^{\alpha}[0,1]$ viewed as a compact set in $C[0,1]$, the $\varepsilon$-entropy satisfies the estimate $\mathcal{H}(\varepsilon)=O\left(\varepsilon^{-1 / \alpha}\right)$. This estimate can be achieved by a simple linear procedure. Say, for $\alpha \leq 1$ it suffices to approximate $u$ by the piecewise-linear function with equidistant nodes. The $\varepsilon$-entropy of the unit ball of $W_{p}^{\alpha}(0,1)$, viewed as a compact set in $L_{p}(0,1)$, satisfies the same estimate. However, nothing was known about the behavior of the $\varepsilon$-entropy in the case where the metric of the superspace and the metric in which the properties of derivatives are described are of different nature, including the simplest case of the embedding $W_{p}^{1}(0,1) \subset C[0,1]$.

This problem was solved in full generality in [38. It was shown that the $\varepsilon$-entropy of the embedding $W_{p}^{l}\left(\mathbb{Q}^{d}\right) \subset L_{q}\left(\mathbb{Q}^{d}\right)$ admits the estimate $\mathcal{H}(\varepsilon)=O\left(\varepsilon^{-d / l}\right)$, provided that the condition $1 / p-l / d<1 / q$ of compactness of this embedding is fulfilled. Thus, the $\varepsilon$-entropy depends only on the order of smoothness, but not on the type of a metric in which this smoothness is measured. It is also important that no linear procedure can achieve the same order of approximation.

\section{§7. Estimates AND ASYMPTOtics OF THE SPECTRUm FOR INTEGRAL AND DIFFERENTIAL OPERATORS}

7.1. Estimates of the spectrum of integral operators. The second field of applications of the method of piecewise-polynomial approximations concerns estimates of singular numbers of integral operators acting in the weighted $L_{2}$-spaces. To obtain such estimates was the initial goal of M. Sh. and M. Z. Solomyak when they started that investigation. As was mentioned in $\S 5$, such estimates were needed for the study of the boundedness of DOI's in the algebra $\mathfrak{B}$ of all bounded linear operators. Methods known at that time were poorly adapted to obtaining estimates of the spectrum of operators in the weighted spaces, and the approach under discussion was invented in order to overcome this obstruction. It should be mentioned that later the same authors have found another, more elementary method of solving these problems.

Main results on the estimates in question were summarized in the paper [69]. That paper contains estimates obtained with the help of piecewise-polynomial approximation, as well as results based on other methods. Ideas of interpolation theory of linear operators were used actively. Though the main purpose was the proof of spectral estimates for operators acting in the weighted spaces, many results turned out to be new also for operators in the "usual" $L_{2}$. Especially, this concerns operators in the entire space $\mathbb{R}^{d}$, when the estimates are determined by interaction between the smoothness of the kernel and its behavior at infinity. For instance, a detailed scale of estimates for the operators with the kernel $b(x) e^{i x \xi} a(\xi)$ was obtained. Such kernels arise in many applications, including the Schrödinger operator and pseudodifferential operators.

7.2. Estimates and asymptotics of the spectrum for "nonsmooth" elliptic problems. The results on piecewise-polynomial approximations directly imply sharporder estimates of the spectrum of "weighted" boundary value problems in arbitrary bounded domains $\Omega \subset \mathbb{R}^{d}$, such as the problem

$$
-\Delta u=\lambda V(x) u,\left.\quad u\right|_{\partial \Omega}=0,
$$

or its analog for the polyharmonic operator. Let $N_{+}(\lambda)$ and $N_{-}(\lambda)$ be the counting functions of positive and negative eigenvalues of the problem (7.1); here the weight 
function $V(x)$ may be sign-indefinite. The asymptotic behavior of $N_{ \pm}(\lambda)$ is given by the classical Weyl-type formula

$$
\lim _{\lambda \rightarrow \infty} \lambda^{-d / 2} N_{ \pm}(\lambda)=c_{0}(d) \int_{\Omega} V_{ \pm}^{d / 2} d x, \quad c_{0}(d)=(\Gamma(d / 2+1))^{-1}(2 \sqrt{\pi})^{-d} .
$$

For $V \equiv 1$ formula (7.2) was obtained by H. Weyl in 1912 by the variational approach. The Tauberian technique developed by Carleman in 1936 turned out to be more flexible, and since then most of the results on spectral asymptotics were obtained by this approach. However, this technique always requires some smoothness assumptions on the boundary and the weight function $V(x)$. By the end of 1960s, for the nonsmooth case nothing was known, except for the results of Weyl and Courant, where formula (7.2) was justified for $V \equiv 1$ and for bounded domains with "not too bad" boundary.

The estimates for $N_{ \pm}(\lambda)$ found by the piecewise-polynomial approximation technique involve the $L_{p}$-norm of $V$ with $p$ depending on dimension $d$. Such estimates turn out to be a powerful tool for the study of spectral asymptotics. The way to apply them is based on an important (though, rather elementary) statement from abstract perturbation theory proved in [49]. As applied to the spectral estimates, this statement implies that the nonlinear functionals

$$
V \mapsto \limsup _{\lambda \rightarrow \infty} \lambda^{-d / 2} N_{ \pm}(\lambda), \quad V \mapsto \liminf _{\lambda \rightarrow \infty} \lambda^{-d / 2} N_{ \pm}(\lambda)
$$

for the problem (7.1) are continuous on $L_{p}$. It is important that this is an a priori fact independent of the specific form of the asymptotic coefficient, which is given in this particular case by (7.2). Application of this general fact and its analogs lies in the foundation of a new version of the variational approach, which was developed by M. Sh. and M. Z. Solomyak in a number of papers starting with [49] and summarized in the lectures 62 (the book B3] in the list of books). This approach turned out to be adequate for a wide class of nonsmooth spectral problems.

7.3. Here we list some results obtained by the approach in question.

1. The asymptotic formula (7.2) for the Dirichlet problem (7.1) is valid in an arbitrary bounded domain $\Omega \subset \mathbb{R}^{d}$ if $V(x)$ is a real-valued function such that $V \in L_{1}(\Omega)$ for $d=1$ and $V \in L_{p}(\Omega)$ with some $p>d / 2$ for $d \geq 2$. Later G. V. Rozenblum showed that in the case where $d \geq 3$ the result is valid if $V \in L_{d / 2}(\Omega)$ in any (not necessarily bounded) domain.

In particular, the initial Weyl formula (i.e., formula (7.2) with $V=1$ ) is valid in any bounded domain of arbitrary dimension. This fact was extended by G. V. Rozenblum to the case of an arbitrary open set of finite measure.

Note that, in the framework of the approach suggested, one encounters no additional difficulties when considering sign indefinite weight functions $V$. Before, only some particular results (by Plejel) were known for the indefinite case.

2. The Weyl-Carleman asymptotic formula was justified in the case of the Dirichlet problem for any uniformly elliptic second order divergence type operator with measurable bounded coefficients in an arbitrary bounded domain (see [57]). In the case of the Neumann boundary condition, the same result was obtained in domains with Lipschitz boundary. In all results of this type known before, some regularity (at least, continuity) of the principal coefficients had been required. Similar results were obtained for operators of higher order, including elliptic systems (see 60]).

3. The spectral asymptotics for integral operators with "weakly polar" kernels acting in $L_{2}(\Omega)$ was obtained in [48. In [67, 73], the spectral asymptotics for 
pseudodifferential operators of negative order under minimal assumptions on the smoothness of the symbol was found.

4. In [75] and a series of subsequent papers, estimates and asymptotics of the spectrum were obtained for the operator associated with a variational problem considered on a space of solutions of a given elliptic system. For instance, such problems arise when one studies the difference of the inverses for one and the same elliptic operator with different boundary conditions, such as $\Delta_{\mathcal{N}}^{-1}-\Delta_{\mathcal{D}}^{-1}$, where $\Delta_{\mathcal{N}}, \Delta_{\mathcal{D}}$ are the Neumann and Dirichlet Laplacians. So, these results can be viewed as a refinement of the estimates found in $[22$.

\section{§8. MaXWell operator in DOMAins With NONSMOOTH BOUNDARY: ASYMPTOTICS OF THE SPECTRUM}

8.1. For an empty electromagnetic resonator in a bounded domain with a smooth ideally conducting boundary, the asymptotic behavior of eigenfrequences was found by H. Weyl in 1912. In the nonsmooth situation (for the filled resonator with nonsmooth boundary and nonsmooth tensors of electric permittivity $\varepsilon(x)$ and magnetic permeability $\mu(x))$ the results were obtained not long ago, mostly, by M. Sh. Birman and his colleagues and students. The reasons of this delay were not purely technical; they lie in the nature of the problem.

Formally, the stationary Maxwell operator is given by the differential expression

$$
M_{\varepsilon, \mu}\{\mathbf{u}, \mathbf{v}\}=\left\{i \varepsilon^{-1} \operatorname{curl} \mathbf{u},-i \mu^{-1} \operatorname{curl} \mathbf{v}\right\}
$$

under the divergence free conditions

$$
\operatorname{div}(\varepsilon \mathbf{u})=0, \quad \operatorname{div}(\mu \mathbf{v})=0
$$

and the boundary conditions

$$
\left.\mathbf{u}_{\tau}\right|_{\partial \Omega}=0,\left.\quad(\mu \mathbf{v})_{\nu}\right|_{\partial \Omega}=0 .
$$

Here $\mathbf{u}$ and $\mathbf{v}$ stand for the electric and magnetic components of the electromagnetic field, the indices $\tau$ and $\nu$ mark the tangential and normal components of a vector field on the boundary. However, it is necessary to define all these operations in a suitable way, but it is not simple to do this if the boundary and the matrix-valued functions $\varepsilon(x), \mu(x)$ are not smooth. Such nonsmoothness is important for many applications, for instance, for the study of electromagnetic oscillations in domains with edges, screens or conic points, and also for the study of the electromagnetic field in stratified media.

The first problem is to define $M_{\varepsilon, \mu}$ as a selfadjoint operator acting in an appropriate Hilbert space. In the case of the filled resonator, this space is the $L_{2}$-space of vectorvalued functions with the matrix-valued weight generated by the matrices $\varepsilon(x), \mu(x)$. Difficulties arise already in the description of the domain of the operator $M_{\varepsilon, \mu}$. The problem is that this operator is not semibounded, and therefore, the quadratic form approach is impossible. The right choice of a selfadjoint realization is dictated by the condition of finiteness for the electromagnetic energy. In the nonsmooth case, the domain of the operator $M_{\varepsilon, \mu}$ cannot be described in terms of Sobolev spaces.

The problem of constructing the "right" selfadjoint realization of the Maxwell operator was solved basically in the papers by N. Weck and R. Picard in 1970-1980s. They described the physically preferable selfadjoint realization of the Maxwell operator by constructing function spaces (in terms of the vector analysis operations) that contain the electric and magnetic components of electromagnetic field. However, these authors did not study the asymptotic behavior of eigenfrequences of electromagnetic oscillations. 
Standard calculations with the operator symbols formally give the following result. Let $r(\xi)$ be the symbol of curl, i.e.

$$
r(\xi)=i\left(\begin{array}{ccc}
0 & \xi_{3} & -\xi_{2} \\
-\xi_{3} & 0 & \xi_{1} \\
\xi_{2} & -\xi_{1} & 0
\end{array}\right)
$$

By $\Lambda_{1}(x, \xi), \Lambda_{2}(x, \xi)$ we denote the positive eigenvalues of the algebraic problem

$$
r(\xi) \mu(x)^{-1} r(\xi) \mathbf{h}=\Lambda \varepsilon(x) \mathbf{h}, \quad \mathbf{h} \in \mathbb{C}^{3}, x \in \Omega, \xi \in \mathbb{R}^{3} ;
$$

the third eigenvalue is equal to zero. We put

$$
\Gamma(\varepsilon, \mu, \Omega)=\frac{1}{24 \pi^{3}} \int_{\Omega} \int_{|\xi|=1}\left(\Lambda_{1}(x, \xi)^{-3 / 2}+\Lambda_{2}(x, \xi)^{-3 / 2}\right) d S(\xi) d x
$$

(here $d S(\xi)$ is the surface element on the unit sphere). Then the eigenfrequencies $m_{k}$ of electromagnetic oscillations (the eigenvalues of the operator $M_{\varepsilon, \mu}$ ) satisfy the following asymptotic formula:

$$
\lim _{k \rightarrow \infty} k^{-1} m_{k}^{3}=\Gamma(\varepsilon, \mu, \Omega) .
$$

The proof of this formula is relatively simple in the case of smooth boundary and smooth matrix-valued functions $\varepsilon(x), \mu(x)$. In the nonsmooth case, the proof requires big additional efforts.

8.2. First papers of M. Sh. Birman on this subject were written in the mid-1970s. In [66. 70, jointly with his student A. B. Alekseev, he developed a general geometric method (in the sense of Hilbert space geometry), which reduces the problem of electromagnetic oscillations of the filled resonator to the simplest case of the empty resonator. In the case of smooth boundary, this directly implies relation (8.1) for any fillings; it is required only that the matrix-valued functions $\varepsilon(x), \mu(x)$ be measurable and bounded together with their inverses. The case of nonsmooth boundary turned out to be more difficult. Further progress was reached with the help of the results about the analytic structure of the vector fields from the domain of the electric component of electromagnetic field. This analysis was carried out in the papers [88, 89, 95] by M. Sh. Birman and in his papers [90, 97] joint with M. Z. Solomyak; see also the survey [93]. Namely, it was shown that the electric component $\mathbf{u}(x)$ in the case of an empty resonator with Lipschitz boundary admits decomposition of the form

$$
\mathbf{u}=\mathbf{u}_{0}+\nabla w,
$$

where $\mathbf{u}_{0} \in \stackrel{\circ}{H}^{1}(\Omega)$, and $w$ is the weak solution of the Dirichlet problem for the Poisson equation: $-\Delta w=f,\left.w\right|_{\partial \Omega}=0$, for some $f \in L_{2}(\Omega)$. A similar decomposition was obtained also for domains with screens. With the help of the decomposition (8.2), in 91] relation (8.1) was proved for an empty resonator with Lipschitz boundary. A final result in this direction was obtained by M. Sh. and his student N. D. Filonov in [160, where the Weyl asymptotics (8.1) was justified in the case of an arbitrary domain $\Omega$ under the only condition that the decomposition (8.2) is valid. This result is of principal importance because it reduces the justification of the asymptotics (8.1) to the purely analytic problem of description of singularities for the vector fields in a certain class. The result for the empty resonator is carried over to the case of the filled resonator automatically by using the method of Birman and Alekseev mentioned above; this method was further developed in the paper [157] joint with Filonov.

The program of the study of spectral characteristics of the "nonsmooth" Maxwell operator was initiated by M. Sh. in the early 1970s. The paper [160] written in 2007 can be viewed as a final work in a series of papers devoted to the realization of this program. 
8.3. It is impossible not to mention the program paper [114, where M. Sh. compared the solvability properties of three important problems in three-dimensional polyhedra: the systems of Maxwell, Stokes, and Lamé. Under apropriate boundary conditions coming from physics, all three of these problems reduce formally to one and the same boundaryvalue problem for the system of first order equations. However, for nonconvex polyhedra the physical origin of these problems dictates a different choice of the selfadjoint realizations for each of them. The paper contains a detailed discussion of this striking effect.

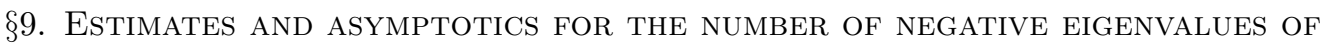 THE SCHRÖDINGER OPERATOR}

9.1. The first estimate for the number of negative eigenvalues of the Schrödinger operator $-\Delta-V$ in $L_{2}\left(\mathbb{R}^{d}\right)$, namely, estimate (2.3), was obtained by M. Sh. in [15, 19. Another important estimate was proved by G. V. Rozenblum in 1972 by refining the piecewise-polynomial approximations approach described above in $\S 6$. This result was later independently proved by E. Lieb and M. Cwikel and is known as the "RozenblumLieb-Cwikel estimate". It has the form

$$
N_{-}(-\Delta-\alpha V) \leq C(d) \alpha^{d / 2} \int_{\mathbb{R}^{d}} V_{+}^{d / 2} d x, \quad \alpha>0, \quad d \geq 3 .
$$

In addition to (9.1), the following asymptotic formula

$$
\lim _{\alpha \rightarrow \infty} \alpha^{-d / 2} N_{-}(-\Delta-\alpha V)=c_{0}(d) \int_{\mathbb{R}^{d}} V_{+}^{d / 2} d x, \quad d \geq 3,
$$

is valid, where $c_{0}(d)$ is the same constant as in (7.2). Estimate (9.1) and the asymptotics (9.2) are valid if the integral on the right-hand side is finite. Moreover, in the case where $d \geq 3$ and $V(x) \geq 0$, the property $V \in L_{d / 2}\left(\mathbb{R}^{d}\right)$ is necessary and sufficient for the quasiclassical estimate $N_{-}(-\Delta-\alpha V)=O\left(\alpha^{d / 2}\right)$.

9.2. For $d \geq 3$, the case where $V \in L_{d / 2}\left(\mathbb{R}^{d}\right)$ is called regular. The irregular case is also possible if the potential $V$ vanishes at infinity (so that the negative spectrum is discrete), but $V \notin L_{d / 2}\left(\mathbb{R}^{d}\right)$. Effects appearing in this case were investigated in detail in a series of papers by M. Sh. Birman and M. Z. Solomyak, the most important of which are [109, 113]. The main tool was linear interpolation; the problem was reduced to it after applying the Birman-Schwinger principle. The base of interpolation was given by estimate (9.1) and an important result of V. G. Maz'ya which gives the boundedness criterion for the embedding of the homogeneous Sobolev space $\mathcal{H}^{l}\left(\mathbb{R}^{d}\right)$ in the weighted space $L_{2, V}\left(\mathbb{R}^{d}\right)$. As a result, a scale of estimates that describe potentials, such that

$$
N_{-}(-\Delta-\alpha V)=O\left(\alpha^{q}\right)
$$

for any given $q>d / 2$, was obtained. In particular, it was shown that sharp results of this type must be formulated in terms of the "weak" weighted $L_{q}$-classes. Estimates given in terms of the "usual" $L_{q}$-classes obtained earlier by Yu. V. Egorov and V. A. Kondrat'ev turned out to be not sharp in the sense that under their assumptions one always has " $O$ " instead of " $O$ " in (9.3). In [109, for any $q>d / 2$ examples of the potentials $V$ such that $N_{-}(-\Delta-\alpha V) \sim C \alpha^{q}$ with $C>0$ were constructed.

It was also proved that in the irregular case the asymptotic behavior of the function $N_{-}(-\Delta-\alpha V)$ as $\alpha \rightarrow \infty$ is determined by the behavior of the potential near its singular points and at infinity. This is in contrast with the regular case: formula (9.2) shows that for $V(x)>0$ any set in $\mathbb{R}^{d}$ with nonzero measure contributes to the asymptotic coefficient. 
Another peculiarity of the irregular estimates is their instability with respect to an additional spectral parameter: it may happen that the function $N_{-}(-\Delta-\alpha V)$ behaves irregularly, but at the same time $N_{-}(-\Delta+h-\alpha V)=O\left(\alpha^{d / 2}\right)$ for any $h>0$.

9.3. In this range of problems, the case of small dimensions $(d=1,2)$ is specific. Estimate (9.1) for $d=1,2$ is not true. In the case of $d=1$, it is natural to consider the operator $H_{\alpha V}=-\frac{d^{2}}{d x^{2}}-\alpha V$ on the semiaxis $\mathbb{R}_{+}$under the Dirichlet condition at the point $x=0$. For this case, first estimates of the quasiclassical order $O\left(\alpha^{1 / 2}\right)$ were obtained by M. Sh. and his student V. V. Borzov in the paper [54 in 1971. Note that the known Bargman estimate for this operator gives $N_{-}\left(H_{\alpha V}\right)=O(\alpha)$ and is irregular in the sense in question. In 109, a more general sufficient condition of regular growth for the function $N_{-}\left(H_{\alpha V}\right)$ was obtained. Next, by interpolation, classes of potentials such that $N_{-}\left(H_{\alpha V}\right)=O\left(\alpha^{q}\right)$ with a given $q>1 / 2$ were found. It was also shown that the corresponding conditions on the potential are not only sufficient, but also necessary. Thus, the one-dimensional case is different from the case of $d \geq 3$, where only sufficient conditions for the behavior $N_{-}(-\Delta-\alpha V)=O\left(\alpha^{q}\right), 2 q>d$, are known (though for the regular behavior the necessary and sufficient condition $V \in L_{d / 2}\left(\mathbb{R}^{d}\right)$ is known).

The results of [109] concern not only the Schrödinger operator, but also its analog $(-\Delta)^{l}-\alpha V$ of higher order. For $2 l<d$, the analog of estimate (9.1) with $d / 2$ replaced by $d / 2 l$ is true. For $2 l>d$ estimates are of different nature; a particular case is the one-dimensional problem discussed above.

The "intermediate" case of $d=2$ studied in the paper [130] by M. Sh. Birman and A. A. Laptev is specific in many respects. In particular, no criterion of the regular behavior $N_{-}(-\Delta-\alpha V)=O(\alpha)$ is known until now, though wide sufficient conditions of such behavior are known. Furthermore, if $d \geq 3$ and $V \geq 0$, then the estimate $N_{-}(-\Delta+h-\alpha V)=O\left(\alpha^{d / 2}\right)$ for some $h>0$ implies the same estimate with $h=0$, whence $V \in L_{d / 2}\left(\mathbb{R}^{d}\right)$ and the asymptotics (9.2) is true. In [130], it was shown that these facts may be violated for $d=2$. Examples of potentials with the following property were constructed: the function $N_{-}(-\Delta+h-\alpha V)$ satisfies the Weyl asymptotics for $h>0$, but for $h=0$ this is not true. The case where $N_{-}(-\Delta-\alpha V)=O\left(\alpha^{q}\right)$ with $q>1$ is possible, and the case where $N_{-}(-\Delta-\alpha V) \sim C \alpha$ with the non-Weyl coefficient $C$ is also possible. The reasons of such effects were clarified: they lie in the structure of the spectrum of the unperturbed operator (the free Laplacian) near the bottom of its spectrum, i.e., in this case, near the point $\lambda=0$. Later M. Sh. and his colleagues found many other manifestations of such threshold effects; see $\S 10$ below.

\section{§10. Discrete SPECTRUm of A PERTURbed operator IN THE SPECTRAL GAPS OF THE UNPERTURBED ONE}

10.1. Suppose that there are gaps in the essential spectrum of a given selfadjoint operator $A$. Typical examples are the Dirac operator, whose spectrum coincides with $(-\infty,-1] \cup[1, \infty)$, and the Schrödinger operator with a periodic potential, whose spectrum has a band structure. Let $V$ be a symmetric operator relatively compact with respect to $A$. Then the discrete spectrum of the perturbed operator $A+V$ may appear in the spectral gaps of $A$. The variational technique does not apply to the study of this discrete spectrum in a direct way. It was shown by M. Sh., as early as in [16, 19, how to overcome this difficulty for the Dirac operator. But only in the 1990s he started to study such problems systematically.

Let $\left(\lambda_{-}, \lambda_{+}\right)$be a gap in the spectrum of the operator $A$ (the case of a semiinfinite gap, i.e., $\lambda_{-}=-\infty$, is not excluded). For simplicity, we suppose that $V \geq 0$ and consider the perturbed operator $A(t)=A-t V, t \geq 0$. When $t$ grows, the eigenvalues $\lambda_{k}(t)$ of $A(t)$ 
"spring up" at the right edge $\lambda_{+}$of the gap and move to the left "disappearing" at the left edge of the gap. Fix an observation point $\lambda \in\left[\lambda_{-}, \lambda_{+}\right]$; let $\alpha>0$. Let $N(A, V, \alpha, \lambda)$ denote the number of eigenvalues $\lambda_{k}(t)$ of $A(t)$ that cross the point $\lambda$ as $t$ grows from 0 to $\alpha$. The problem of interest is the asymptotic behavior of $N(A, V, \alpha, \lambda)$ for a fixed $\lambda$ and $\alpha \rightarrow \infty$.

10.2. In a big series of papers [110, 111, 118, 119, 120, 123, 127, 134, 135, 144, 146 , (especially, see the survey [128]), M. Sh. Birman, partly with his colleagues and students, studies this problem in detail. There is an essential difference between the cases where $\lambda \in\left[\lambda_{-}, \lambda_{+}\right)$and $\lambda=\lambda_{+}$(when the observation point coincides with the right edge of the gap). For the first case, a general "comparison theorem" was established in [110]. Let $A$ be semibounded from below, and let $B$ be obtained from $A$ by a form-bounded perturbation with zero bound. The comparison theorem says that, under some assumptions on $V$, the quantities $N(A, V, \alpha, \lambda)$ and $N(B, V, \alpha, \mu)$ have the same asymptotic behavior as $\alpha \rightarrow \infty$, for arbitrary real $\lambda \in \rho(A)$ and $\mu \in \rho(B)$ (here $\rho(\cdot)$ stands for the set of all regular points of the operator under consideration). In applications to the perturbed periodic Schrödinger operator $-\Delta+p(x)-t V(x)$ (when $A=-\Delta, B=-\Delta+p(x)$, and $p(x)$ is a periodic potential) this allows one to use known results on the negative spectrum of the "usual" Schrödinger operator $-\Delta-t V(x)$; see $\S 9$. The case of $\lambda=\lambda_{+}$ is much more difficult; here threshold effects mentioned in $\S 9$ may appear.

10.3. We dwell on the results for the case where the unperturbed operator acts in $L_{2}\left(\mathbb{R}^{d}\right)$ and is given by

$$
H=-\operatorname{div} g(x) \nabla+p(x)
$$

Here $g(x)$ is a positive definite matrix-valued function with real entries and $p(x)$ is a real-valued function; both $g(x)$ and $p(x)$ are periodic with respect to some lattice. For simplicity, we assume that the lattice of periods is $\mathbb{Z}^{d}$. First, we recall what the structure of the spectrum of a periodic operator $H$ is. By the Floquet-Bloch theory, the operator $H$ is expanded (in the sense of unitary equivalence) in the direct integral

$$
H \sim \int_{[-\pi, \pi)^{d}}^{\oplus} H(\mathbf{k}) d \mathbf{k},
$$

where the operators $H(\mathbf{k})$ act in $L_{2}\left(\mathbb{Q}^{d}\right)$ and depend on the parameter $\mathbf{k}$ (the quasimomentum). Recall that $\mathbb{Q}^{d}$ is the unit cube in $\mathbb{R}^{d}$. The operator $H(\mathbf{k})$ is given by the differential expression

$$
H(\mathbf{k})=(\mathbf{D}+\mathbf{k})^{*} g(x)(\mathbf{D}+\mathbf{k})+p(x)
$$

with periodic boundary conditions; here $\mathbf{D}=-i \nabla$. The spectrum of $H(\mathbf{k})$ is discrete; the corresponding consecutive eigenvalues are denoted by $E_{j}(\mathbf{k}), j \in \mathbb{N}$ (they are enumerated with multiplicities taken into account). The corresponding eigenfunctions of the operator $H(\mathbf{k})$ are denoted by $\varphi_{j}(x ; \mathbf{k})$. The eigenvalues $E_{j}(\mathbf{k})$ viewed as functions of the parameter $\mathbf{k}$ are called band functions. The band functions are continuous and $(2 \pi \mathbb{Z})^{d}$-periodic. The spectrum of $H$ has a band structure: it is the union of the closed intervals (spectral bands) that are the ranges of the band functions $E_{j}(\cdot), j \in \mathbb{N}$. Bands may overlap (for $d \geq 2$ ); on the other hand, there may be gaps in the spectrum. Let $\left(\lambda_{-}, \lambda_{+}\right)$be a gap. Then the number $\lambda_{+}$is the minimum of some band function $E_{j}(\cdot)$. Usually, some regularity assumptions about the structure of the edge of the gap are imposed. Namely, it is assumed that there is only one band function $E_{j}$ whose minimum is $\lambda_{+}$, this minimum is attained only at a finite number of points, and $E_{j}$ has quadratic behavior near these minimum points. For the bottom of the spectrum, these conditions are valid automatically. By the threshold characteristics of the operator $H$ at the edge 
of the gap $\lambda_{+}$, we mean the asymptotics of the functions $E_{j}(\cdot)$ and $\varphi_{j}(x ; \cdot)$ near the minimum points of $E_{j}$.

10.4. Consider the perturbed operator $H(t)=H-t V$, where $V(x)$ is a real-valued function vanishing as $|x| \rightarrow \infty$. The behavior of the spectrum of $H(t)$ in a gap is similar to that of the negative discrete spectrum of the usual Schrödinger operator. Let $d \geq 3$. Then the case where $V \in L_{d / 2}\left(\mathbb{R}^{d}\right)$ is regular, and $N\left(H, V, \alpha, \lambda_{+}\right)$satisfies the Weyl asymptotics. The case where $V \notin L_{d / 2}\left(\mathbb{R}^{d}\right)$ is irregular; for any $q>d / 2$ one can present a class of potentials $V$ such that $N\left(H, V, \alpha, \lambda_{+}\right) \sim C \alpha^{q}$. Next, the asymptotic coefficient $C$ is determined not only by the potential $V$, but also by the threshold characteristics of $H$ at the edge of the gap. The corresponding asymptotics is called "threshold" asymptotics. For $d \geq 3$, the regular case was studied in [127, and the irregular case in 134 .

Precisely as for the "usual" Schrödinger operator, the two-dimensional case is specific; the spectrum in the semiinfinite gap $\left(-\infty, \lambda_{+}\right)$for the two-dimensional operator $H(t)$ was studied in the paper 144 by M. Sh. Birman, A. A. Laptev, and T. A. Suslina. In this case, asymptotics may be irregular. For any $q>1$ a class of potentials $V$ such that $N\left(H, V, \alpha, \lambda_{+}\right) \sim C \alpha^{q}$ was distinguished; the coefficient $C$ is of threshold nature. Also, a class of potentials $V$ was presented for which a competition between the Weyl and threshold asymptotics occurs: $N\left(H, V, \alpha, \lambda_{+}\right) \sim C \alpha$ (i.e., the asymptotics is of Weyl order), but the asymptotic coefficient is given by the sum of the Weyl and threshold terms.

We mention also the paper [146] by M. Sh. Birman and M. Z. Solomyak, where such effects were observed for the second order operator in a $d$-dimensional waveguide periodic in one direction. It was assumed that the coefficients of the unperturbed operator are also periodic, and the perturbation vanishes as $|x| \rightarrow \infty$ along the waveguide axis. The discrete spectrum of the perturbed operator in the semiinfinite gap was studied. This problem is close to the one-dimensional case, though it is much more difficult. In distinction to the problem in the entire $\mathbb{R}^{d}$, here a threshold effect is present in any dimension $d>1$.

\section{§11. Absolute continuity of the spectrum OF PERIODIC OPERATORS OF MATHEMATICAL PHYSICS}

11.1. As was discussed above in Subsection 10.3, the spectrum of a periodic elliptic operator acting in $L_{2}\left(\mathbb{R}^{d}\right)$ has a band structure. From a theoretical point of view, some spectral bands may degenerate into points, which are eigenvalues of infinite multiplicity. This happens if the corresponding band function is constant: $E_{j}(\mathbf{k})=$ const. (Examples of operators such that this degeneration does exist are known.) If there are no degenerate bands, then the spectrum of a periodic operator is purely absolutely continuous. The problem of the proof of the absence of degenerate spectral bands is important and difficult.

First, the absence of degenerate spectral bands for the periodic Schrödinger operator $H=-\Delta+p(x)$ in $L_{2}\left(\mathbb{R}^{d}\right)$ was proved by $\mathrm{L}$. Thomas in 1973 . We explain the main idea of the Thomas approach. For simplicity, assume that the lattice of periods is $\mathbb{Z}^{d}$. Let $H(\mathbf{k})$ be the family of operators in $L_{2}\left(\mathbb{Q}^{d}\right)$ arising in the direct integral expansion for $H$ (see (10.1), (10.2) with $g=1$ ). This is an analytic operator family with compact resolvent. The Thomas approach consists in the study of the analytic extension of the operator family $H(\mathbf{k})$ to complex values of the quasimomentum. Precisely, we put $\mathbf{k}=\left(\pi+i y, \mathbf{k}^{\prime}\right)$, where $\mathbf{k}^{\prime} \in \mathbb{R}^{d-1}$ is fixed and $y=\operatorname{Im} k_{1}$ is a large parameter. Thomas showed that the corresponding operator $H(y):=H\left(\pi+i y, \mathbf{k}^{\prime}\right)$ is invertible for sufficiently large $|y|$. 
Moreover, the Thomas estimate

$$
\left\|H(y)^{-1}\right\| \leq C(1+|y|)^{-1}, \quad|y| \geq y_{0},
$$

is valid. By the analytic Fredholm alternative, this easily implies the absence of degenerate spectral bands.

First, estimate (11.1) is proved (with the help of the Fourier series) for the "free" operator $H_{0}(y)$ that corresponds to the case of $p(x)=0$. Next, the potential $p(x)$ is taken into account as an additive perturbation, and estimate (11.1) is carried over to the case of the "perturbed" operator $H(y)$.

11.2. The magnetic Schrödinger operator $M=(\mathbf{D}-\mathbf{A}(x))^{2}+p(x)$ is a much more difficult case. Here $\mathbf{A}(x)$ is a periodic vector-valued (magnetic) potential. It is impossible to prove an analog of (11.1) for the corresponding operator $M(y)$ by viewing $M(y)$ as an additive perturbation of the "free" operator $H_{0}(y)$, because the perturbation contains terms of order of $|y|$. This difficulty had not been overcome during almost a quarter of a century.

In 1997 in the breakthrough paper [131], M. Sh. Birman and T. A. Suslina proved the absolute continuity of the spectrum of the periodic magnetic Hamiltonian $M$ in dimension $d=2$. The approach of [131] was based on the study of the operator $P=$ $(\mathbf{D}-\mathbf{A})^{2}+\partial_{1} A_{2}-\partial_{2} A_{1}$ (one of two blocks of the two-dimensional Pauli operator), which admits a convenient factorization. Due to this factorization, it is possible to treat $P(y)$ as a multiplicative perturbation of the operator $H_{0}(y)$ and to prove estimate of the form (11.1) for $P(y)^{-1}$. Next, there is no problem to pass from $P(y)$ to $M(y)$, viewing $M(y)-P(y)$ as an additive perturbation.

After almost 25 years of stagnation, the paper [131] stimulated a series of studies, done by different authors, on the absolute continuity of the spectrum of periodic operators. For $d \geq 3$ the problem of absolute continuity for the spectrum of the periodic magnetic Hamiltonian was solved in 1997 by A. V. Sobolev (the case where $d \geq 3$ turned out to be much more difficult than the two-dimensional case).

M. Sh. Birman himself together with T. A. Suslina continued investigations in this field in the papers [138, 140, 141, 142, 148] (in [142] the third coathor was R. G. Shterenberg, a student of M. Sh.).

\section{§12. ThrESHOLD PROPERTIES AND HOMOGENIZATION PROBLEMS FOR PERIODIC DIFFERENTIAL OPERATORS}

12.1. The last big and important circle of papers by M. Sh. Birman (written jointly with T. A. Suslina), the main of which are 145, 151, 155, 156, 158, 161, is devoted to the study of threshold properties and homogenization problems for periodic differential operators. This series begins with the paper [139, where the spectral characteristics of the two-dimensional periodic Pauli operator at the bottom of the spectrum (the threshold characteristics) were studied. It became clear that some "good" properties of the threshold characteristics are related not only to the specific features of the Pauli operator, but to the existence of factorization of the form $X^{*} X$, where $X$ is a homogeneous differential first order operator. Due to this observation, a wide class of matrix periodic differential operators was singled out (see Subsection 12.2) and the threshold properties of these operators were studied. During this work, an idea was born that the threshold properties of periodic differential operators must be related to homogenization problems in the small period limit. Later it was realized that it is possible and useful to study homogenization procedure for a periodic operator as a spectral threshold effect at the bottom of the spectrum. On this way, new type results in homogenization theory, called the "operator error estimates", were obtained. 
12.2. Let us describe the class of operators. In the space $L_{2}\left(\mathbb{R}^{d} ; \mathbb{C}^{n}\right)$, consider a matrix elliptic second order operator $A$ admitting a factorization of the form

$$
A=A(g, f)=f(x)^{*} b(\mathbf{D})^{*} g(x) b(\mathbf{D}) f(x) .
$$

Here $g(x)$ and $f(x)$ are periodic matrix-valued functions of sizes $m \times m$ and $n \times n$, respectively, where $m \geq n$. Both matrices are bounded together with their inverses, and $g(x)$ is positive definite. Next, $b(\mathbf{D})$ is an $(m \times n)$-matrix homogeneous first order differential operator; its symbol $b(\xi), \xi \in \mathbb{R}^{d}$, is a matrix of maximal rank for all $\xi \neq 0$. In the case where $f(x)$ is the identity matrix, we use the notation $\widehat{A}=\widehat{A}(g)=A(g, \mathbf{1})$. Many periodic operators of mathematical physics admit such a factorization.

12.3. The main objects of study are the operators $\widehat{A}_{\varepsilon}=\widehat{A}\left(g^{\varepsilon}\right), A_{\varepsilon}=A\left(g^{\varepsilon}, f^{\varepsilon}\right), \varepsilon>0$, where $g^{\varepsilon}(x)=g\left(\varepsilon^{-1} x\right), f^{\varepsilon}(x)=f\left(\varepsilon^{-1} x\right)$. The coefficients of these operators oscillate rapidly for small $\varepsilon$. Homogenization theory studies properties of such operators as $\varepsilon \rightarrow 0$.

A typical elliptic homogenization problem is to study the behavior of the solution $u_{\varepsilon}$ of the equation

$$
\widehat{A}_{\varepsilon} u_{\varepsilon}+u_{\varepsilon}=F, \quad F \in L_{2}\left(\mathbb{R}^{d} ; \mathbb{C}^{n}\right),
$$

for small $\varepsilon$. It is known that there exists a limit: $u_{\varepsilon} \rightarrow u_{0}$ as $\varepsilon \rightarrow 0$, where $u_{0}$ is the solution of the so called "homogenized" (or "effective") equation

$$
\widehat{A}^{0} u_{0}+u_{0}=F \text {. }
$$

Here $\widehat{A}^{0}=b(\mathbf{D})^{*} g^{0} b(\mathbf{D})$ is the "effective" operator, and $g^{0}$ is the constant positive "effective" matrix. The way of finding the effective matrix is well known. It is nontrivial: $g^{0}$ is defined in terms of periodic solutions of some auxiliary elliptic problems on the cell of the lattice of periods.

The questions about the character of convergence of $u_{\varepsilon}$ to $u_{0}$ and about error estimate arise. By traditional methods of homogenization theory, the strong convergence in $L_{2}$ with the error estimate $\left\|u_{\varepsilon}-u_{0}\right\|_{L_{2}} \leq C \varepsilon$ was proved. However, it remained unknown how the constant $C$ depends on the right-hand side $F$ of equation (12.2).

In [145, it was shown for the first time that

$$
\left\|u_{\varepsilon}-u_{0}\right\|_{L_{2}} \leq C \varepsilon\|F\|_{L_{2}} .
$$

Estimate (12.4) can be rewritten in operator terms:

$$
\left\|\left(\widehat{A}_{\varepsilon}+I\right)^{-1}-\left(\widehat{A}^{0}+I\right)^{-1}\right\|_{L_{2} \rightarrow L_{2}} \leq C \varepsilon,
$$

that is why such estimates are called "operator error estimates". Estimate (12.5) is order-sharp; the constant $C$ is controlled explicitly. In [151, a similar but somewhat more complicated approximation for the resolvent of the more general operator $A_{\varepsilon}$ was found.

Later in [155, 156, more accurate approximations of the resolvents of $\hat{A}_{\varepsilon}$ and $A_{\varepsilon}$ in the $L_{2}\left(\mathbb{R}^{d} ; \mathbb{C}^{n}\right)$-operator norm with error term $O\left(\varepsilon^{2}\right)$ were obtained. In [158, approximation of the resolvent in the norm of operators acting from $L_{2}\left(\mathbb{R}^{d} ; \mathbb{C}^{n}\right)$ to the Sobolev space $H^{1}\left(\mathbb{R}^{d} ; \mathbb{C}^{n}\right)$ with error term $O(\varepsilon)$ was found. In both cases, it is necessary to take terms of order $\varepsilon$ (the so-called correctors) into account. An interesting effect was discovered: the form of the corrector depends on the type of the operator norm in which an error is measured. 
12.4. General results were applied to the specific operators of mathematical physics. The most difficult case was the homogenization problem for the stationary periodic Maxwell system, which was studied in [151, Chapter 7], [159] and in some papers by T. A. Suslina. The method devepoled first for elliptic problems was later carried over to parabolic homogenization problems by T. A. Suslina.

The last significant result in this field was obtained in the paper 161 by M. Sh. Birman and T. A. Suslina. It concerns the operator error estimates in homogenization problems for nonstationary equations of Schrödinger type and of hyperbolic type. Consider the Cauchy problem for the Schrödinger type equation:

$$
i \frac{\partial u_{\varepsilon}(x, \tau)}{\partial \tau}=\widehat{A}_{\varepsilon} u_{\varepsilon}, \quad u_{\varepsilon}(x, 0)=\phi(x) ; \quad \phi \in L_{2}\left(\mathbb{R}^{d} ; \mathbb{C}^{n}\right) .
$$

For a fixed $\tau$, the solution $u_{\varepsilon}$ converges as $\varepsilon \rightarrow 0$ in $L_{2}\left(\mathbb{R}^{d} ; \mathbb{C}^{n}\right)$ to the solution $u_{0}$ of the homogenized problem:

$$
i \frac{\partial u_{0}(x, \tau)}{\partial \tau}=\widehat{A}^{0} u_{0}, \quad u_{0}(x, 0)=\phi(x)
$$

The problem is to estimate the error term. Here new difficulties arise: it is impossible to estimate the difference of the operator exponentials $\exp \left(-i \widehat{A}_{\varepsilon} \tau\right)-\exp \left(-i \widehat{A}^{0} \tau\right)$ in the $L_{2}$-operator norm. However, it is possible to obtain the estimate

$$
\left\|\exp \left(-i \widehat{A}_{\varepsilon} \tau\right)-\exp \left(-i \widehat{A}^{0} \tau\right)\right\|_{H^{3} \rightarrow L_{2}} \leq\left(C_{1}+C_{2}|\tau|\right) \varepsilon
$$

which makes it possible to prove the qualified error estimates

$$
\left\|u_{\varepsilon}-u_{0}\right\|_{L_{2}} \leq\left(C_{1}+C_{2}|\tau|\right)^{s / 3} \varepsilon^{s / 3}\|\phi\|_{H^{s}}, \quad \phi \in H^{s}\left(\mathbb{R}^{d} ; \mathbb{C}^{n}\right), \quad 0<s \leq 3 .
$$

Similar results were also obtained for the hyperbolic equations.

12.5. Now we explain main ideas of the method, on the example of the proof of estimate (12.5). Again, we assume that the lattice of periods is $\mathbb{Z}^{d}$. By the scaling transformation, (12.5) is reduced to the estimate

$$
\left\|\left(\widehat{A}+\varepsilon^{2} I\right)^{-1}-\left(\hat{A}^{0}+\varepsilon^{2} I\right)^{-1}\right\|_{L_{2} \rightarrow L_{2}} \leq C \varepsilon^{-1} .
$$

Thus, it is required to approximate the resolvent of the operator $\widehat{A}$ at the point $-\varepsilon^{2}$, which is near the bottom of the spectrum $\lambda=0$. Naturally, the main impact in such approximation must be given by the threshold characteristics of $\hat{A}$ at the bottom of the spectrum. The operator $\widehat{A}$ is expanded in the direct integral of operators $\widehat{A}(\mathbf{k})$ acting in $L_{2}\left(\mathbb{Q}^{d}\right)$ (see $\left.(10.1),(10.2)\right)$. Then estimate $(12.6)$ is equivalent to a similar approximation in the $L_{2}\left(\mathbb{Q}^{d}\right)$-operator norm for the resolvent $\left(\widehat{A}(\mathbf{k})+\varepsilon^{2} I\right)^{-1}$ (this approximation must be uniform with respect to the parameter $\left.\mathbf{k} \in[-\pi, \pi)^{d}\right)$. The main part of investigation is the study of the properties of the operator family $\widehat{A}(\mathbf{k})$, which is an analytic operator family with compact resolvent, by methods of the analytic perturbation theory with respect to the one-dimensional parameter $t=|\mathbf{k}|$. On this way, it is possible to construct appropriate approximation of the resolvent in terms of an auxiliary finite rank operator (called the "spectral germ"). This operator is defined in terms of the threshold characteristics of $\hat{A}$. It turns out that the effective operator $\widehat{A}^{0}$ has the same germ; this leads to the required results.

Thus, the method discussed above shows that the homogenization procedure is a manifestation of the threshold effect at the bottom of the spectrum of the operator $\widehat{A}$. 


\section{§13. CONCLUding REMARKS}

In conclusion, the authors would like to share with the reader some general impressions about the scientific style of M. Sh. Birman, collected during long time contacts with him.

One of the most important qualities of M. Sh. was the strategic character of his mathematical thought. He was rarely thinking in terms of concrete problems, however interesting and important they might be in themselves. He always tried to reveal and clarify main principles that determine the general nature of possible results in a range of problems he was working on. The following way of thinking was typical for him: first a general approach, which covers the problem (or problems) in question, is constructed, next this approach is analyzed exhaustively. As a result, a theory appears applicable to a wide class of problems of a given type. Finally, it is shown how the objects of the general theory are realized in the concrete case under consideration. As a rule, this way leads to an exhaustive analysis not merely of the initial particular problem, but of the entire class of related questions. This approach has manifested itself brightly already in his work on extension theory (see $\S 2$ of this survey). The same concerns the work of M. Sh. on the spectrum of singular boundary value problems. One of later examples is the new approach to homogenization theory (see $\S 12$ ).

M. Sh. realized this feature of himself very well. One of us told him once about answering a seemingly inapproachable question. His reply was:

- You have resolved a question? Very good! And, probably, I am doomed forever to resolve general problems.

What was said does not mean that M. Sh. was weaker in solving specific problems. His papers on scattering theory and also on applications of the quadratic forms technique in the study of boundary value problems are full of technical tricks, which were later used widely by many mathematicians and physicists. Each of us can give many examples when, during joint work with M. Sh., an obstacle arising was overcome with the help of some technical tool suggested by him in one of his earlier papers. In such cases, he liked to cite Koz'ma Prutkov:

"Wait, my charming, my temptress,

Soon or may be later,

I'll pull out from my dress

A silky-smooth rope-ladder".

M. Sh. had a wonderful ability to see a general problem beyond a particular example, and to see an idea or, moreover, a general ideology beyond a technical tool. A bright example of first type is his paper [25] on the Laplace operator in domains with edges. An example of second type is related to his paper [38, where the piecewise-polynomial approximation method was developed. As was mentioned in $\S 7$, the initial goal of the authors was to find conditions under which a given integral operator in the weighted $L_{2}$-space belongs to the trace class. Paying attention to the nonlinear version of the method, M. Sh. supposed that it could help to estimate the $\varepsilon$-entropy of the embeddings of Sobolev classes in the spaces $C$ and $L_{q}$. This was confirmed brilliantly.

An exceptional independence of thought was typical of M. Sh.. He felt free with introducing new notions. Let us give an example. During a long time it was common to describe spectral properties of compact operators in terms of the Schatten-von Neumann classes, though in many important questions such a description was evidently not sharp. In the paper [22, M. Sh. introduced classes which are now called the "weak Schatten-von Neumann classes". This allowed him to obtain spectral estimates for the problem studied in 22 in sharp terms. Another example (from the late work) is the introduction of the new term "spectral germ", which plays a crucial role in the description of the threshold properties of a periodic elliptic operator. 
M. Sh. was inclined to self-analysis. When he obtained a new scientific result, he always asked himself: why have I managed to do this and why haven't others? This was not because of self-advertising: he tried to understand what combination of experience and knowledge helped him to solve the problem.

He was always moving forward, though he did not neglect working out an investigation started before. M. Sh. told: "During all my life I am writing one and the same paper", though he brought significant contributions to different fields of mathematical physics, function theory, spectral theory of operators, partial differential equations. But, when he passed from one subject to another, one could always trace the threads which connected these subjects with each other. In this survey, the authors tried to mention these threads. He had a wonderful intuition. He felt faultlessly what deserved to be studied and what did not. Sometimes, he felt the relationship between different fields and subjects under investigation on some irrational level.

At the same time, his mentality was not purely mathematical. His choice of subjects for investigation was often motivated by direct applications to natural physical problems. He had many talents, and mathematics was lucky to be his favourite.

He was indifferent to the honoured titles and rewards, and always considered that it was beneath his dignity to strive to get them. However, in the declining years, when he was seriously ill, we was glad to be awarded the titles of the Honoured Scientist of Russian Federation, the Honoured Professor of St. Petersburg State University, and the Chebyshev Prize. These rewards showed the (belated) recognition of his services.

Due to his sharp intellect, wide erudition, and fast reaction on everything around him, M. Sh. was a leader in any group which he joined. It was always interesting and instructive to listen to him.

The memory of Mikhail Shlemovich Birman will always live in the hearts of those who were lucky to know this wonderful scientist and person.

\section{List of PUblications of M. Sh. Birman}

\section{PAPERS}

[1] M. Sh. Birman, Some estimates for the method of steepest descent, Uspekhi Mat. Nauk (N.S.) 5 (1950), no. 3, 152-155; English transl., NBS Rep. 2007; Four Articles on Numerical Matrix Methods, U.S. Dep. Commerc., Nat. Bureau Standards, Washington, DC, 1952, pp. 51-56. MR0035922 (12:32d) MR0050985(14:412d)

[2] On computation of eigenvalues by the method of steepest descent, Zap. Leningrad. Gornogo Inst. 27 (1952), no. 1, 209-216. (Russian)

[3] _ On a variant of the method of successive approximations, Vestnik Leningrad. Univ. Ser. Mat. Phys. Chem. 1952, no. 9, 69-76. (Russian)

[4] _ On the theory of selfadjoint extensions of positive definite operators, Dokl. Akad. Nauk SSSR (N.S.) 91 (1953), no. 2, 189-191. (Russian) MR0058130(15:326f)

[5] _ On the theory of general boundary problems for elliptic differential equations, Dokl. Akad. Nauk SSSR (N.S.) 92 (1953), no. 2, 205-208. (Russian) MR0062922 (16:42e)

[6] _ On minimal functionals for elliptic differential equations of second order, Dokl. Akad. Nauk SSSR (N.S.) 93 (1953), no. 6, 953-956. (Russian) MR0062923 (16:42f)

[7] _ On the spectrum of singular boundary problems for elliptic differential equations, Dokl. Akad. Nauk SSSR (N.S.) 97 (1954), no. 1, 5-7. (Russian) MR.0064283 (16:258e)

[8] , On Trefftz's variational method for the equation $\Delta^{2} u=f$, Dokl. Akad. Nauk SSSR (N.S.) 101 (1955), no. 2, 201-204. (Russian) MR0070831(17:41e)

[9] _ On the Friedrichs extension procedure of a positive operator to the selfadjoint one, Zap. Leningrad. Gornogo Inst. 33 (1956), no. 3, 132-136.

[10] $\quad$ On the theory of selfadjoint extensions of positive definite operators, Mat. Sb. (N.S.) 38 (80) (1956), no. 4, 431-450. (Russian) MR0080271 (18:220d)

[11] Variational methods of solution of boundary problems analogous to the method of Trefftz, Vestnik Leningrad. Univ. Ser. Mat. Mekh. Astronom. 1956, vyp. 3, 69-89. (Russian) MR0080867 $(18: 316 f)$ 
[12] _ Method of quadratic forms in problems with small parameter at the highest derivatives, Vestnik Leningrad. Univ. Ser. Mat. Mekh. Astronom. 1957, vyp. 3, 9-12. (Russian) MR0101398 $(21: 209)$

[13] A characterization of elliptic differential operators with maximal domain, Vestnik Leningrad. Univ. Ser. Mat. Mekh. Astronom. 1957, vyp. 4, 177-183. (Russian)

[14] - On multi-dimensional boundary value problems with a small parameter at higher derivatives, Uspekhi Mat. Nauk (N.S.) 12 (1957), no. 6, 212-213. (Russian)

[15] - Perturbations of quadratic forms and the spectrum of singular boundary value problems, Dokl. Akad. Nauk SSSR 125 (1959), no. 3, 471-474. (Russian) MR0107816 (21:6538)

[16] _ On the spectrum of Schrödinger and Dirac operators, Dokl. Akad. Nauk SSSR 129 (1959), no. 2, 239-241. (Russian) MR0109934 (22:817)

[17] - On the discrete part of the spectrum of Schrödinger and Dirac operators, Vestnik Leningrad. Univ. Ser. Mat. Mekh. Astronom. 1960, vyp. 2, 167-168. (Russian)

[18] M. Sh. Birman and B. S. Pavlov, On the complete continuity of certain imbedding operators, Vestnik Leningrad. Univ. Ser. Mat. Mekh. Astronom. 1961, vyp. 1, 61-74. (Russian) MR0133491 (24:A3322)

[19] M. Sh. Birman, On the spectrum of singular boundary-value-problems, Mat. Sb. (N.S.) 55 (1961), no. 2, 125-174; English transl., 11 Papers on Analysis, Amer. Math. Soc. Transl.(2), vol. 53, Amer. Math. Soc., Providence, RI, 1966, pp. 23-60. MR0142896 (26:463) MR.0194283 (33:2496)

[20] - Perturbation of the spectrum of a singular elliptic operator under variation of the boundary and boundary conditions, Dokl. Akad. Nauk SSSR 137 (1961), no. 4, 761-763; English transl., Soviet Math. Dokl. 2 (1961), 326-328. MR0177311 (31:1574)

[21] _ On the number of eigenvalues in a quantum scattering problem, Vestnik Leningrad. Univ. Ser. Mat. Mekh. Astronom. 1961, vyp. 3, 163-166. (Russian) MR0139370 (25:2803)

[22] - Perturbations of the continuous spectrum of a singular elliptic operator by varying the boundary and the boundary conditions, Vestnik Leningrad. Univ. Ser. Mat. Mekh. Astronom. 1962, vyp. 1, 22-55; English transl., Amer. Math. Soc. Transl., Ser. 2 (2008), vol. 225, 19-53. MR.0138874 $(25: 2314)$

[23] - On existence conditions for wave operators, Dokl. Akad. Nauk SSSR 143 (1962), no. 3, 506-509; English transl., Soviet Math. Dokl. 3 (1962), 408-411. MR0149333 (26:6823)

[24] M. Sh. Birman and M. G. KreĬn, On the theory of wave operators and scattering operators, Dokl. Akad. Nauk SSSR 144 (1962), no. 3, 475-478; English transl., Soviet Math. Dokl. 3 (1962), 740744. MR 0139007 (25:2447)

[25] M. Sh. Birman and G. E. Skvortsov, On square summability of highest derivatives of the solution of the Dirichlet problem in a domain with piecewise smooth boundary, Izv. Vyssh. Uchebn. Zaved. Mat. 1962, no. 5, 12-21. (Russian) MR0145196 (26:2731)

[26] M. Sh. Birman, On a test for the existence of wave operators, Dokl. Akad. Nauk SSSR 147 (1962), no. 5, 1008-1009; English transl., Soviet Math. Dokl. 3 (1962), 1747-1748. MR0167841(29:5107)

[27] _ Existence conditions for wave operators, Izv. Akad. Nauk SSSR Ser. Mat. 27 (1963), no. 4, 883-906. (Russian) MR0161150 (28:4359)

[28] M. Sh. Birman and M. G. Krĕn, Some topics of the theory of the wave and scattering operators, 1963 Outlines Joint Sympos. Partial Differential Equations (Novosibirsk, 1963), Akad. Nauk SSSR Sibirsk. Otdel., Moscow, 1963, pp. 39-45. MR0200749 (34:637)

[29] M. Sh. Birman and I. M. Glazman, Spectra of singular differential operators, Proc. Fourth AllUnion Math. Congr. (Leningrad, 1961). Vol. II, Nauka, Leningrad, 1964, pp. 253-261. (Russian) MR0220101(36:3168)

[30] M. Sh. Birman and S. B. Ėntina, A stationary approach in the abstract theory of scattering, Dokl. Akad. Nauk SSSR 155 (1964), no. 3, 506-508; English transl., Soviet Math. Dokl. 5 (1965), 432-435. MR0164590 (29:1887)

[31] M. Sh. Birman, A local criterion for the existence of wave operators, Dokl. Akad. Nauk SSSR 159 (1964), no. 3, 485-488; English transl., Soviet Math. Dokl. 5 (1965), no. 2, 1505-1509. MR0173964 $(30: 4171)$

[32] M. Sh. Birman and M. Z. Solomyak, On double Stieltjes operator integrals, Dokl. Akad. Nauk SSSR 165 (1965), no. 6, 1223-1226; English transl., Soviet Math. Dokl. 6 (1965), no. 2, 15671571. MR0188802 (32:6234)

[33] , Double Stieltjes operator integrals, Spectral Theory and Wave Processes, Probl. Mat. Fiz., No. 1, Leningrad. Univ., Leningrad, 1966, pp. 33-67; English transl., Topics in Math. Phys., vol. 1, Consultants Bureau Plenum Publ. Corporation, New York, 1967, pp. 25-54. MR0209872 (35:767b) MR0210536(35:1428) 
[34] _ Approximation of functions of the $W_{p}^{\alpha}$-classes by piecewise-polynomial functions, Dokl. Akad. Nauk SSSR 171 (1966), no. 5, 1015-1018; English transl., Soviet Math. Dokl. 7 (1966), no. 2, 1573-1577. MR0209733(35:630)

[35] _ Double Stieltjes operator integrals and problems on multipliers, Dokl. Akad. Nauk SSSR 171 (1966), no. 6, 1251-1254; English transl., Soviet Math. Dokl. 7 (1966), no. 2, 1618-1621. MR.0209871(35:767a)

[36] M. Sh. Birman and S. B. Èntina, Stationary approach in abstract scattering theory, Izv. Akad. Nauk SSSR Ser. Mat. 31 (1967), no. 2, 401-430; English transl., Math. USSR-Izv. 1 (1967), no. 1, 391-420. MR0209895 (35:790)

[37] M. Sh. Birman and M. Z. Solomyak, Double Stieltjes operator integrals. II, Spectral Theory, Diffraction Problems, Probl. Mat. Fiz., No. 2, Leningrad. Univ., Leningrad, 1967, pp. 26-60; English transl., Topics in Math. Phys., vol. 2, Consultants Bureau, New York, 1968, pp. 19-46. MR0234304 $(38: 2621)$ MR 0266727 (42:1630)

[38] - Piecewise polynomial approximations of functions of classes $W_{p}^{\alpha}$, Mat. Sb. (N.S.) 73 (115) (1967), no. 3, 331-355; English transl., Math. USSR-Sb. 2 (1967), 295-317. MR0217487 $(36: 576)$

[39] Estimates of singular numbers of integral operators. I, Vestnik Leningrad. Univ. Ser. Mat. Mekh. Astronom. 1967, vyp. 2, 43-53. (Russian) MR0216339 (35:7173)

[40] _ Estimates of singular numbers of integral operators. II, Vestnik Leningrad. Univ. Ser. Mat. Mekh. Astronom. 1967, vyp. 3, 21-28. (Russian) MR0217650 (36:739)

[41] M. Sh. Birman, A local test for the existence of wave operators, Izv. Akad. Nauk SSSR Ser. Mat. 32 (1968), no. 4, 914-942; English transl., Math. USSR-Izv. 2 (1968), no. 2, 879-906. MR0248558 $(40: 1810)$

[42] A. L. Belopol'skiu and M. Sh. Birman, Existence of wave operators in scattering theory for a pair of spaces, Izv. Akad. Nauk SSSR Ser. Mat. 32 (1968), no. 5, 1162-1175; English transl., Math. USSR-Izv. 2 (1968), no. 2, 1117-1130. MR0238101 (38:6377)

[43] M. Sh. Birman and M. Z. Solomyak, Estimates of singular numbers of integral operators. III. Operators in unbounded domains, Vestnik Leningrad. Univ. Ser. Mat. Mekh. Astronom. 1969, vyp. 1, 35-48. (Russian) MR0246163(39:7468)

[44] M. Sh. Birman, Some applications of a local criterion for the existence of wave operators, Dokl. Akad. Nauk SSSR 185 (1969), no. 4, 735-738; English transl., Soviet Math. Dokl. 10 (1969), 393-397. MR0282242(43:7954)

[45] M. Sh. Birman and M. Z. Solomyak, Remarks on the nuclearity of integral operators and the boundedness of pseudodifferential operators, Izv. Vyssh. Uchebn. Zaved. Mat. 1969, no. 9, 11-17. (Russian) MR0254670 (40:7877)

[46] M. Sh. Birman, Scattering problems for differential operators with constant coefficients, Funktsional. Anal. i Prilozhen. 3 (1969), no. 3, 1-16; English transl., Funct. Anal. Appl. 3 (1969), no. 3, 167-180. MR0253083(40:6298)

[47] A test of the existence of complete wave operators in scattering theory for a pair of spaces, Spectral Theory. Wave Processes, Probl. Mat. Fiz., No. 4, Leningrad. Univ., Leningrad, 1970, pp. 22-26; English transl., Topics in Math. Phys., vol. 4, Consultants Bureau, New YorkLondon, 1971, pp. 17-21. MR0276817(43:2557). MR0339975 (49:4732)

[48] M. Sh. Birman and M. Z. Solomyak, Asymptotics of the spectrum of weakly polar integral operators, Izv. Akad. Nauk SSSR Ser. Mat. 34 (1970), no. 5, 1142-1158; English transl., Math. USSR-Izv. 4 (1970), 1151-1168 (1971). MR0279638 (43:5359)

[49] _ The principal term of the spectral asymptotics for "nonsmooth" elliptic problems, Funktsional. Anal. i Prilozhen. 4 (1970), no. 4, 1-13; English transl., Funct. Anal. Appl. 4 (1970), 265-275 (1971). MR0278126 (43:3857)

[50] - On the proof of the theorem on the asymptotic behavior of the spectrum for the real part of a Volterra operator with nuclear imaginary component, Mat. Issled. 5 (1970), no. 4, 16-25. (Russian) MR0284851 (44:2075)

[51] M. Sh. Birman, Works on spectral theory of differential operators, Mathematics in PetersburgLeningrad Univ., Leningrad. Univ., Leningrad, 1970, pp. 129-133. (Russian)

[52] _ Scattering problems for differential operators under a perturbation of the space, Izv. Akad.Nauk SSSR Ser. Mat. 35 (1971), no. 2, 440-455; English transl., Math. USSR-Izv. 5 (1971), 459-474 (1972). MR0291868 (45:957)

[53] M. Sh. Birman and M. Z. Solomyak, The asymptotics of the spectrum of "nonsmooth" elliptic equations, Funktsional. Anal. i Prilozhen. 5 (1971), no. 1, 69-70; English transl., Funct. Anal. Appl. 5 (1971), 56-57. MR0283428(44:659) 
[54] M. Sh. Birman and V. V. Borzov, The asymptotic behavior of the discrete spectrum of certain singular differential operators, Spectral Theory, Probl. Mat. Fiz., No. 5, Leningrad. Univ., Leningrad, 1971, pp. 24-38; English transl., Topics in Math. Phys., vol. 5, Consultants Bureau, New YorkLondon, 1972, pp. 19-30. MR0301568 (46:726), MR0339976 (49:4733)

[55] M. Sh. Birman and M. Z. Solomyak, Remarks on the spectral shift function, Zap. Nauchn. Sem. Leningrad. Otdel. Mat. Inst. Steklov. (LOMI) 27 (1972), 33-46; English transl., J. Soviet Math. 3 (1975), no. 4, 408-419. MR0315482 (47:4031)

[56] _ Spectral asymptotic behavior of nonsmooth elliptic operators, Dokl. Akad. Nauk SSSR 205 (1972), no. 2, 267-270; English transl., Soviet Math. Dokl. 13 (1972), 906-910. MR0305148 (46:4278)

[57] Spectral asymptotics of nonsmooth elliptic operators. I, Trudy Moskov. Mat. Obshch. 27 (1972), 3-52; English transl., Trans. Moscow Math. Soc. 27 (1972), 1-52 (1975). MR0364898 $(51: 1152)$

[58] V. M. Babich, M. Sh. Birman, V. I. Smirnov, M. Z. Solomyak, and N. N. Ural'tseva, Ol'ga Aleksandrovna Ladyzhenskaya (on her fiftieth birthday), Vestnik Leningrad. Univ. Ser. Mat. Mekh. Astronom. 1972, vyp. 2, 159-160. (Russian) MR0297506 (45:6560)

[59] M. Sh. Birman and M. Z. Solomyak, Double Stieltjes operator integrals. III, Theory of Functions. Spectral Theory. Wave Propagation, Probl. Mat. Fiz., No. 6, Leningrad. Univ., Leningrad, 1973, pp. 27-53. (Russian) MR0348494 (50:992)

[60] Spectral asymptotics of nonsmooth elliptic operators. II, Trudy Moskov. Mat. Obshch. 28 (1973), 3-34; English transl., Trans. Moscow Math. Soc. 28 (1973), 1-32 (1975). MR0364898 $(51: 1152)$

[61] Equivalent renormings of $W_{p}^{\alpha}$ classes in the case of partitioning of the domain, Izv. Vyssh. Uchebn. Zaved. 1973, no. 3, 19-27. (Russian) MR0344875 (49:9614)

[62] - Quantitative analysis in Sobolev's imbedding theorems and applications to spectral theory, Tenth Mathematical School (Summer School, Kaciveli/Nal'chik, 1972), Inst. Mat. Akad. Nauk Ukrain. SSR, Kiev, 1974, pp. 5-189. (Russian) MR0482138(58:2224)

[63] _ A certain "model" nonelliptic spectral problem, Collection of Articles Dedicated to the Memory of Academician V. I. Smirnov, Vestnik Leningrad. Univ. Ser. Mat. Mekh. Astronom. 1975, vyp. 1, 39-45; English transl., Vestnik Leningrad. Univ. Math. 8 (1980), 23-30. MR0380148 $(52: 1049)$

[64] M. Sh. Birman, L. S. Koplienko, and M. Z. Solomyak, Estimates of the spectrum of a difference of fractional powers of selfadjoint operators, Izv. Vyssh. Uchebn. Zaved. Mat. 1975, no. 3, 3-10; English transl., Soviet Math. (Iz. VUZ) 19 (1975), no. 3, 1-6 (1976). MR0385597 (52:6458)

[65] A. B. Alekseev and M. Sh. Birman, Asymptotic behavior of the spectrum of elliptic boundary value problems with solvable constraints, Dokl. Akad. Nauk SSSR 230 (1976), no. 3, 505-507; English transl., Soviet Math. Dokl. 17 (1976), no. 5, 1319-1322. MR0420027 (54:8044)

[66] A variational formulation of the problem of the oscillations of a resonator that is filled with a stratified anisotropic medium, Vestnik Leningrad. Univ. Ser. Mat. Mekh. Astronom. 1977, vyp. 2, 9-15; English transl., Vestnik Leningrad. Univ. Math. 10 (1982), 101-108. MR0462202 $(57: 2177)$

[67] M. Sh. Birman and M. Z. Solomyak, Asymptotic behavior of the spectrum of pseudodifferential operators with anisotropically homogeneous symbols, Vestnik Leningrad. Univ. Ser. Mat. Mekh. Astronom. 1977, vyp. 3, 13-21; English transl., Vestnik Leningrad Univ. Math. 10 (1982), 237247. MR0463738 (57:3680)

[68] Application of interpolational methods to estimates of the spectrum of integral operators, Operator Theory in Function Spaces (Proc. School, Novosibirsk, 1975), Nauka, Sibirsk. Otdel., Novosibirsk, 1977, pp. 42-70; English transl., Amer. Math. Soc. Transl. Ser. 2, vol. 127, Amer.Math. Soc., Providence, RI, 1985, pp. 61-82. MR0502834 (80b:47063)

[69] _ Estimates for the singular numbers of integral operators, Uspekhi Mat. Nauk 32 (1977), no. 1, 17-84; English transl., Russian Math. Surveys 32 (1977), no. 1, 15-89. MR0438186 $(55: 11104)$

[70] A. B. Alekseev and M. Sh. Birman, Asymptotic behavior of the discrete spectrum of elliptic problems with solvable differential constraints, Uspekhi Mat. Nauk 32 (1977), no. 1, 232-233. (Russian)

[71] M. Sh. Birman and M. Z. Solomyak, Asymptotic properties of the spectrum of differential equations, Itogi Nauki i Tekhniki Mat. Anal., vol. 14, VINITI, Moscow, 1977, pp. 5-58; English transl., J. Soviet Math. 12 (1979), no. 3, 247-283. MR0467022 (57:6892)

[72] _ On asymptotic behavior of the spectrum of elliptic variational problems with constraints, Proc.All-Union Conf. on Partial Differential Equations Dedicated to Acad. Petrovskiu 75th Birthday, Moskov. Univ., Moscow, 1978, pp. 51-52. (Russian) 
[73] _ Asymptotic behavior of the spectrum of pseudodifferential operators with anisotropically homogeneous symbols. II, Vestnik Leningrad. Univ. Ser. Mat. Mekh. Astronom. 1979, vyp. 3, 5-10; English transl., Vestnik Leningrad Univ. Math. 12 (1980), 155-161. MR0555971 (81b:47060)

[74] M. Sh. Birman, A. M. Vershik, and M. Z. Solomyak, The product of commuting spectral measures may fail to be countably additive, Funktsional. Anal. i Prilozhen. 13 (1979), no. 1, 61-62; English transl., Funct. Anal. Appl. 13 (1979), no. 1, 48-49. MR.0527523 (80h:28010)

[75] M. Sh. Birman and M. Z. Solomyak, Asymptotic behavior of the spectrum of variational problems on solutions of elliptic equations, Sibirsk. Mat. Zh. 20 (1979), no. 1, 3-22; English transl., Siberian Math. J. 20 (1979), no. 1, 1-15. MR0523133 (80c:35077)

[76] _ Asymptotic behavior of the spectrum of variational problems on solutions of elliptic equations, Partial Differential Equations (Proc. Conf., Novosibirsk, 1978), Nauka, Sibirsk. Otdel., Novosibirsk, 1980, pp. 221-223. (Russian) MR0601449 (82b:35115)

[77] _ Asymptotic behavior of the spectrum of variational problems on solutions of elliptic equations in unbounded domains, Funktsional. Anal. i Prilozhen. 14 (1980), no. 4, 27-35; English transl., Funct. Anal. Appl. 14 (1980), 267-274. MR0595726 (82a:35084)

[78] M. Sh. Birman and D. R. Yafaev, Asymptotics of the spectrum of the s-matrix in potential scattering, Dokl. Akad. Nauk SSSR 255 (1980), no. 5, 1085-1087. (Russian) MR0613721 (82j:35116)

[79] M. Sh. Birman, Quasiclassical spectral asymptotic behavior of a class of integral operators, Zap. Nauchn. Sem. Leningrad. Otdel. Mat. Inst. Steklov. (LOMI) 98 (1980), 22-32; English transl., J. Soviet Math. 21 (1983), 12-20. MR0591859 (82i:47083)

[80] M. Sh. Birman and D. R. Yafaev, Asymptotic behavior of the spectrum of the scattering matrix, Zap. Nauchn. Sem. Leningrad. Otdel. Mat. Inst. Steklov. (LOMI) 110 (1981), 3-29; English transl., J. Soviet Math. 25 (1984), 793-814. MR0643971 (83c:35095)

[81] _ Asymptotic behavior of limit phases for scattering by potentials without spherical symmetry, Teoret. Mat. Fiz. 51 (1982), no. 1, 44-53; English transl., Theoret. and Math. Phys. 51 (1982), no. 1, 344-350. MR.0672765 (84a:81037)

[82] M. Sh. Birman and M. Z. Solomyak, The asymptotic behavior of the spectrum of variational problems on solutions of elliptic systems, Zap. Nauchn. Sem. Leningrad. Otdel. Mat. Inst. Steklov. (LOMI) 115 (1982), 23-39; English transl., J. Soviet Math. 28 (1985), 633-644. MR0660069 $(84 \mathrm{c}: 35082)$

[83] - Asymptotic behavior of the spectrum of pseudodifferential variational problems with constraints, Spectral Theory. Wave Processes, Probl. Mat. Fiz., No. 10, Leningrad. Univ., Leningrad, 1982, pp. 20-36; English transl., Selecta Math. Sov. 5 (1986), 245-256. MR0691142 (84f:35109)

[84] - On subspaces that admit a pseudodifferential projector, Vestnik Leningrad. Univ. Ser. Mat. Mekh. Astronom. 1982, vyp. 1, 18-25; English transl., Vestnik Leningrad Univ. Math. 15 (1983), 17-27. MR0652058 (83h:58091)

[85] _ Compact operators with power asymptotic behavior of the singular numbers, Zap. Nauchn. Sem. Leningrad. Otdel. Mat. Inst. Steklov. (LOMI) 126 (1983), 21-30; English transl., J. Soviet Math. 27 (1984), 2442-2447. MR0697420 (85e:47027)

[86] M. Sh. Birman, Re-expansion operators as objects of spectral analysis, Linear and Complex Analysis Problem Book. 199 Research Problems, Lecture Notes in Math., vol. 1043, Springer-Verlag, Berlin, 1984, pp. 130-134. MR0734178 (85k:46001)

[87] M. Sh. Birman and M. Z. Solomyak, Some problems on compact operators with power-like behaviour of singular numbers, Linear and Complex Analysis Problem Book. 199 Research Problems, Lecture Notes in Math., vol. 1043, Springer-Verlag, Berlin, 1984, pp. 217-218. MR0734178 (85k:46001)

[88] M. Sh. Birman, The Maxwell operator in domains with edges, Zap. Nauchn. Sem. Leningrad. Otdel. Mat. Inst. Steklov. (LOMI) 147 (1985), 3-9; English transl., J. Soviet Math. 37 (1987), no. 1, 793-797. MR0821471 (87j:35307)

[89] _ The Maxwell operator for a resonator with inward edges, Vestnik Leningrad. Univ. Ser. 1 1986, vyp. 3, 3-8; English transl., Vestnik Leningrad Univ. Math. 19 (1986), no. 3, 1-8. MR0867387 (87m:35181)

[90] M. Sh. Birman and M. Z. Solomyak, The Maxwell operator in domains with a nonsmooth boundary, Sibirsk. Mat. Zh. 28 (1987), no. 1, 23-36; English transl., Siberian Math. J. 28 (1987), $12-24$. MR0886850 (88g:35095)

[91] Weyl asymptotics of the spectrum of the Maxwell operator for domains with a Lipschitz boundary, Vestnik Leningrad. Univ. Ser. 1 1987, vyp. 3, 23-28; English transl., Vestnik Leningrad Univ. Math. 20 (1987), no. 3, 15-21. MR.0928156(89h:35253) 
[92] _ Construction in a piecewise-smooth domain of a function of the class $H^{2}$ from the value of the conormal derivative, Zap. Nauchn. Sem. Leningrad. Otdel. Mat. Inst. Steklov. (LOMI) 163 (1987), 17-28; English transl., J. Soviet Math. 49 (1990), no. 5, 1128-1136. MR0918938 (89b:35035)

[93],$L_{2}$-theory of the Maxwell operator in arbitrary domains, Uspekhi Mat. Nauk 42 (1987), no. 6, 61-76; English transl., Russian Math. Surveys 42 (1987), no. 6, 75-96. MR0933995 $(89 \mathrm{e}: 35127)$

[94] M. Sh. Birman and D. R. Yafaev, A general scheme in the stationary theory of scattering, Wave Propagation. Scattering Theory, Probl. Mat. Fiz., No. 12, Leningrad. Univ., Leningrad, 1987, pp. 89-117; English transl., Amer. Math. Soc. Transl. (2), vol. 157, Amer. Math. Soc., Providence, RI, 1993, pp. 87-112. MR0923973 (89b:47016), MR1252226

[95] M. Sh. Birman, The Maxwell operator for a periodic resonator with inward edges, Trudy Mat. Inst. Akad. Nauk SSSR 179 (1988), 23-35; English transl., Proc. Steklov Inst. Math. 1989, no. 2, 21-34. MR0964911 (89m:35160)

[96] M. Sh. Birman and M. Z. Solomyak, The $L_{2}$-theory of the Maxwell operator in domains with nonsmooth boundary, Symposium "Partial Differential Equations" (Holzhau, 1988), Teubner-Texte Math., vol. 112, Teubner, Leipzig, 1989, pp. 33-41. MR.1105797

[97] _ The selfadjoint Maxwell operator in arbitrary domains, Algebra i Analiz 1 (1989), no. 1, 96-110; English transl., Leningrad Math. J. 1 (1990), no. 1, 99-115. MR1015335 (91e:35197)

[98] _ Interpolation estimates for the number of negative eigenvalues of a Schrödinger operator, Schrödinger Operators, Standard and Nonstandard (Dubna, 1988), World Sci. Publishing, Teaneck, NJ, 1989, pp. 2-18. MR 1091987 (92e:35115)

[99] M. Sh. Birman and D. R. Yafaev, On the trace-class method in potential scattering theory, Zap. Nauchn. Sem. Leningrad. Otdel. Mat. Inst. Steklov. (LOMI) 171 (1989), 12-35; English transl., J. Soviet Math. 56 (1991), no. 2, 2285-2299. MR1031982 (91g:35198)

[100] M. Sh. Birman and M. Z. Solomyak, Operator integration, perturbations and commutators, Zap. Nauchn. Sem. Leningrad. Otdel. Mat. Inst. Steklov. (LOMI) 170 (1989), 34-66; English transl., J. Soviet Math. 63 (1993), no. 2, 129-148. MR1039579 (91b:47086)

[101] Estimates for the difference of fractional powers of selfadjoint operators under unbounded perturbations, Zap. Nauchn. Sem. Leningrad. Otdel. Mat. Inst. Steklov. (LOMI) 178 (1989), 120145; English transl., J. Soviet Math. 61 (1992), no. 2, 2018-2035. MR1037767 (91d:47006)

[102] _ Negative discrete spectrum of the Schrödinger operator with large coupling constant: a qualitative discussion, Order, Disorder and Chaos in Quantum Systems (Dubna, 1989), Oper. Theory Adv. Appl., vol. 46, Birkhäuser, Basel, 1990, pp. 3-16. MR1124648 (92j:35136)

[103] M. Sh. Birman, Discrete spectrum in the gaps of the continuous one in the large-coupling-constant limit, Order, Disorder and Chaos in Quantum Systems (Dubna, 1989), Oper. Theory Adv. Appl., vol. 46, Birkhäuser, Basel, 1990, pp. 17-25. MR1124649 (92j:47091)

[104] M. Sh. Birman and M. Z. Solomyak, The estimates for the number of negative bound states of the Schrödinger operator for large coupling constants, Integral Equations and Inverse Problems (Varna, 1989), Pitman Res. Notes Math. Ser., vol. 235, Longman Sci. Tech., Harlow, 1991, pp. 49-57. MR 1117952 (92g:35159)

[105] M. Sh. Birman, S. G. Krĕn, O. A. Ladyzhenskaya, G. B. Rozenblum, and Yu. G. Safarov, Mikhail Zakharovich Solomyak (on the occasion of his sixtieth birthday), Uspekhi Mat. Nauk 46 (1991), no. 4, 183-184; English transl., Russian Math. Surveys 46 (1991), no. 4, 217-219. MR.1138976 (92k:01025)

[106] M. Sh. Birman, On the discrete spectrum in gaps of a second-order perturbed periodic operator, Funktsional.Anal. i Prilozhen. 25 (1991), no. 2, 89-92; English transl., Funct. Anal. Appl. 25 (1991), no. 2, 158-161. MR1142222 (92m:47090)

[107] _ Discrete spectrum in a gap of perturbed periodic operator at large coupling constants, Rigorous Results in Quantum Dynamics (Liblice, 1990), World Sci. Publishing, River Edge, NJ, 1991, pp. 16-24. MR1243026

[108] M. Sh. Birman and M. Z. Solomyak, Discrete negative spectrum under nonregular perturbations (polyharmonic operators, Schrödinger operators with a magnetic field, periodic operators), Rigorous Results in Quantum Dynamics (Liblice, 1990), World Sci. Publishing, River Edge, NJ, 1991, pp. 25-36. MR 1243027

[109] Estimates for the number of negative eigenvalues of the Schrödinger operator and its generalizations, Estimates and Asymptotics for Discrete Spectra of Integral and Differential Equations (Leningrad, 1989-90), Adv. Soviet Math., vol. 7, Amer. Math. Soc., Providence, RI, 1991, pp. 1-55. MR1306507 (95h:35161) 
[110] M. Sh. Birman, Discrete spectrum in the gaps of a continuous one for perturbations with large coupling constant, Estimates and Asymptotics for Discrete Spectra of Integral and Differential Equations (Leningrad, 1989-90), Adv. Soviet Math., vol. 7, Amer. Math. Soc., Providence, RI, 1991, pp. 57-73. MR1306508 (95h:47009)

[111] M. Sh. Birman and G. D. Raikov, Discrete spectrum in the gaps for perturbations of the magnetic Schrödinger operator, Estimates and Asymptotics for Discrete Spectra of Integral and Differential Equations (Leningrad, 1989-90), Adv. Soviet Math., vol. 7, Amer. Math. Soc., Providence, RI, 1991, pp. 75-84. MR1306509 (95h:35153)

[112] M. Sh. Birman, G. E. Karadzhov, and M. Z. Solomyak, Boundedness conditions and spectrum estimates for the operators $b(X) a(D)$ and their analogs, Estimates and Asymptotics for Discrete Spectra of Integral and Differential Equations (Leningrad, 1989-90), Adv. Soviet Math., vol. 7, Amer. Math. Soc., Providence, RI, 1991, pp. 85-106. MR1306510 (95g:47075)

[113] M. Sh. Birman and M. Z. Solomyak, Schrödinger operator. Estimates for number of bound states as function-theoretical problem, Spectral Theory of Operators (Novgorod, 1989), Amer. Math. Soc. Transl. Ser. 2, vol. 150, Amer. Math. Soc., Providence, RI, 1992, pp. 1-54. MR1157648(93j:35120)

[114] M. Sh. Birman, Three problems in continuum theory in polyhedra, Zap. Nauchn. Sem. S.-Peterburg. Otdel. Mat. Inst. Steklov. (POMI) 200 (1992), 27-37; English transl., J. Math. Sci. (N.Y.) 77 (1995), no. 3, 3153-3160. MR1192110 (94b:35204)

[115] M. Sh. Birman and D. R. Yafaev, The spectral shift function. The papers of M. G. Kreŭn and their further development, Algebra i Analiz 4 (1992), no. 5, 1-44; English transl., St. Petersburg Math. J. 4 (1993), no. 5, 833-870. MR.1202723 (94g:47002)

[116] _ Spectral properties of the scattering matrix, Algebra i Analiz 4 (1992), no. 6, 1-27; English transl., St. Petersburg Math. J. 4 (1993), no. 6, 1055-1079. MR1199632 (94g:47003)

[117] M. Sh. Birman and M. Z. Solomyak, On the main singularities of the electric component of the electro-magnetic field in regions with screens, Algebra i Analiz 5 (1993), no. 1, 143-159; English transl., St. Petersburg Math. J. 5 (1994), no. 1, 125-139. MR.1220492 (94f:35137)

[118] M. Sh. Birman, Discrete spectrum of the periodic Schrödinger operator for non-negative perturbations, Mathematical Results in Quantum Mechanics (Blossin, 1993), Oper. Theory Adv. Appl., vol. 70, Birkhäuser, Basel, 1994, pp. 3-7. MR.1308998

[119] M. Sh. Birman and T. Weidl, The discrete spectrum in a gap of the continuous one for compact supported perturbations, Mathematical Results in Quantum Mechanics (Blossin, 1993), Oper. Theory Adv. Appl., vol. 70, Birkhäuser, Basel, 1994, pp. 9-12. MR1308999

[120] M. Sh. Birman and A. Laptev, Discrete spectrum of the perturbed Dirac operator, Mathematical Results in Quantum Mechanics (Blossin, 1993), Oper. Theory Adv. Appl., vol. 70, Birkhäuser, Basel, 1994, pp. 55-59. MR1309006

[121] M. Sh. Birman and D. R. Yafaev, Scattering matrix for the perturbation of a periodic Schrödinger operator by a decaying potential, Algebra i Analiz 6 (1994), no. 3, 17-39; English transl., St. Petersburg Math. J. 6 (1995), no. 3, 453-474. MR1301828(95i:47086)

[122] M. Sh. Birman, Discrete spectrum of the periodic elliptic operator with a differential perturbation, Journées "Équations aux Dérivées Partielles" (Saint-Jean-de-Monts, 1994), Exp. No. XIV, École Polytech., Palaiseau, 1994, 4 pp. MR 1298685

[123] M. Sh. Birman and A. Laptev, Discrete spectrum of the perturbed Dirac operator, Ark. Mat. 32 (1994), no. 1, 13-32. MR1277918(95h:35162)

[124] M. Sh. Birman, Ilya Bakelman in Russia. A tribute to Ilya Bakelman (College Station, TX, 1993), Discourses Math. Appl., vol. 3, Texas A \& M Univ., College Station, TX, 1994, pp. 2-4. MR.1423361

[125] V. I. Arnol'd, M. Sh. Birman, I. M. Gel'fand et al., Anatoliǔ Moiseevich Vershik (on the occasion of his sixtieth birthday), Uspekhi Mat. Nauk 49 (1994), no. 3, 195-204; English transl., Russian Math. Surveys 49 (1994), no. 3, 207-221. MR1289402

[126] M. Sh. Birman, Spectral shift function and double operator integrals, Linear and Complex Analysis Problem Book 3. Part I, Lecture Notes in Math., vol. 1573, Springer-Verlag, Berlin, 1994, pp. 272273. MR 1334345 (96c:00001a)

[127] _ , The discrete spectrum in gaps of the perturbed periodic Schrödinger operator. I. Regular perturbations, Boundary Value Problems, Schrödinger Operators, Deformation Quantization, Math. Top., vol. 8, Akademie Verlag, Berlin, 1995, pp. 334-352. MR.1389015 (97d:47055)

[128] Discrete spectrum of the periodic Schrödinger operator perturbed by a decaying potential, Algebra i Analiz 8 (1996), no. 1, 3-20; English transl., St. Petersburg Math. J. 8 (1997), no. 1, 1-14. MR:1392011 (97h:47047) 
[129] M. Sh. Birman and M. Solomyak, Tensor product of a finite number of spectral measures is always a spectral measure, Integral Equations Operator Theory 24 (1996), no. 2, 179-187. MR.1371945 (96m:47038)

[130] M. Sh. Birman and A. Laptev, The negative discrete spectrum of a two-dimensional Schrödinger operator, Comm. Pure Appl. Math. 49 (1996), no. 9, 967-997. MR1399202 (97i:35131)

[131] M. Sh. Birman and T. A. Suslina, Two-dimensional periodic magnetic Hamiltonian is absolutely continuous, Algera i Analiz 9 (1997), no. 1, 32-48; English transl., St. Petersburg Math. J. 9 (1998), no. 1, 21-32. MR 1458417 (98g:47038)

[132] M. Sh. Birman, A. Laptev, and M. Solomyak, The negative discrete spectrum of the operator $(-\Delta)^{l}-\alpha V$ in $L_{2}\left(\mathbf{R}^{d}\right)$ for $d$ even and $2 l \geq d$, Ark. Mat. 35 (1997), no. 1, 87-126. MR1443037 (98d:35167)

[133] M. Sh. Birman and A. Laptev, "Non-standard" spectral asymptotics for a two-dimensional Schrödinger operator, Partial Differential Equations and their Applications (Toronto, ON, 1995), CRM Proc. Lecture Notes, vol. 12, Amer. Math. Soc., Providence, RI, 1997, pp. 9-16. MR1479234

[134] M. Sh. Birman, Discrete spectrum in the gaps of a perturbed periodic Schrödinger operator. II. Nonregular perturbations, Algebra i Analiz 9 (1997), no. 6, 62-89; English transl., St. Petersburg Math. J. 9 (1998), no. 6, 1073-1095. MR1610239 (99h:47054)

[135] M. Sh. Birman and A. B. Pushnitskiı̌, Discrete spectrum in the gaps of the perturbed pseudorelativistic magnetic Hamiltonian, Zap. Nauchn. Sem. S.-Peterburg. Otdel. Mat. Inst. Steklov.(POMI) 249 (1997), 102-117; English transl., J. Math. Sci. (N.Y.) 101 (2000), no. 5, 3437-3447. MR1698515 (2000h:35123)

[136] Spectral shift function, amazing and multifaceted, Integral Equations Operator Theory 30 (1998), no. 2, 191-199. MR1607900 (98m:47012)

[137] M. Sh. Birman, A. Laptev, and M. Solomyak, On the eigenvalue behaviour for a class of differential operators on semiaxis, Math. Nachr. 195 (1998), 17-46. MR1654744 (99m:34194)

[138] M. Sh. Birman and T. A. Suslina, Absolute continuity of the two-dimensional periodic magnetic Hamiltonian with discontinuous vector-valued potential, Algebra i Analiz 10 (1998), no. 4, 1-36; English transl., St. Petersburg Math. J. 10 (1999), no. 4, 579-601. MR.1654063 (99k:81060)

[139] _ Two-dimensional periodic Pauli operator. The effective masses at the lower edge of the spectrum, Mathematical Results in Quantum Mechanics (Prague, 1998), Oper. Theory Adv. Appl., vol. 108, Birkhäuser, Basel, 1999, pp. 13-31. MR1708785 (2000g:81049)

[140] - The periodic Dirac operator is absolutely continuous, Integral Equations Operator Theory 34 (1999), no. 4, 377-395. MR1702229 (2000h:47068)

[141] Periodic magnetic Hamiltonian with variable metric. The problem of absolute continuity, Algebra i Analiz 11 (1999), no. 2, 1-40; English transl., St. Petersburg Math. J. 11 (2000), no. 2, 203-232. MR1702587 (2000i:35026)

[142] M. Sh. Birman, T. A. Suslina, and R. G. Shterenberg, Absolute continuity of the spectrum of a two-dimensional Schrödinger operator with potential supported on a periodic system of curves, Algebra i Analiz 12 (2000), no. 6, 140-177; English transl., St. Petersburg Math. J. 12 (2001), no. 6, 983-1012. MR 1816514 (2002k:35227)

[143] M. Sh. Birman and T. A. Suslina, On the absolute continuity of the periodic Schrödinger and Dirac operators with magnetic potential, Differential Equations and Mathematical Physics (Birmingham, AL, 1999), AMS/IP Stud. Adv. Math., vol. 16, Amer. Math. Soc., Providence, RI, 2000, pp. 41-49. MR 1764740

[144] M. Sh. Birman, A. Laptev, and T. A. Suslina, Discrete spectrum of a two-dimensional periodic elliptic second order operator perturbed by a decaying potential. I. Semibounded gap, Algebra i Analiz 12 (2000), no. 4, 36-78; English transl., St. Petersburg Math. J. 12 (2001), no. 4, 535-567. MR1793617 (2003b:47078)

[145] M. Sh. Birman and T. A. Suslina, Threshold effects near the lower edge of the spectrum for periodic differential operators of mathematical physics, Systems, Approximation, Singular Integral Operators, and Related Topics (Bordeaux, 2000), Oper. Theory Adv. Appl., vol. 129, Birkhäuser, Basel, 2001, pp. 71-107. MR.1882692 (2003f:35220)

[146] M. Sh. Birman and M. Z. Solomyak, On the negative discrete spectrum of a periodic elliptic operator in a waveguide-type domain, perturbed by a decaying potential, J. Anal. Math. 83 (2001), 337-391. MR 1828497 (2002k:35226)

[147] B. A. Amosov, M. Sh. Birman, M. I. Vishik et al., Mikhail Semenovich Agranovich (on the occasion of his seventieth birthday), Uspekhi Mat. Nauk 56 (2001), no. 4, 163-168; English transl., Russian Math. Surveys 56 (2001), no. 4, 777-784. MR1861459(2002h:01026) 
[148] M. Sh. Birman and T. A. Suslina, Absolute continuity of the spectrum of the periodic operator of elasticity theory for constant shear modulus, Nonlinear Problems in Mathematical Physics and Related Topics, II, Mezhdunar. Mat. Ser., vol. 2, Tamara Rozhkovskaya, Novosibirsk, 2002, pp. 6570; English transl., Int. Math. Ser., vol. 2, Kluwer/Plenum, New York etc., 2002, pp. 69-74. MR $1971990(2004 c: 35296)$

[149] A. A. Arkhipova, M. Sh. Birman, V. S. Buslaev et al., On the jubilee of Ol'ga Aleksandrovna Ladyzhenskaya, Zap. Nauchn. Sem. S.-Peterburg. Otdel. Mat. Inst. Steklov. (POMI) 288 (2002), 5-13; English transl., J. Math. Sci. (N.Y.) 123 (2004), no. 6, 4523-4526. MR.1923542

[150] M. Sh. Birman, On homogenization procedure for periodic operators near the edge of an internal gap, Algebra i Analiz 15 (2003), no. 4, 61-71; English transl., St. Petersburg Math. J. 15 (2004), no. 4, 507-513. MR2068979(2006i:35010)

[151] M. Sh. Birman and T. A. Suslina, Second order periodic differential operators. Threshold properties and homogenization, Algebra i Analiz 15 (2003), no. 5, 1-108; English transl., St. Petersburg Math. J. 15 (2004), no. 5, 639-714. MR2068790 (2005k:47097)

[152] M. Sh. Birman and M. Z. Solomyak, Double operator integrals in a Hilbert space, Integral Equations Operator Theory 47 (2003), 131-168. MR2002663 (2004f:47029)

[153] M. Sh. Birman and T. A. Suslina, Homogenization of multidimensional periodic elliptic operator in a neighborhood of an edge of an inner gap, Zap. Nauchn. Sem. S.-Peterburg. Otdel. Mat. Inst. Steklov. (POMI) 318 (2004), 60-74; English transl., J. Math. Sci. (N.Y.) 136 (2006), no. 2, 36823690. MR2120232(2005j:35006)

[154] V. I. Arnol'd, M. Sh. Birman, A. M. Vershik et al., Ol'ga Aleksandrovna Ladyzhenskaya, Uspekhi Mat. Nauk 59 (2004), no. 3, 151-152; English transl., Russian Math. Surveys 59 (2004), no. 3, 553-555. MR2116538

[155] M. Sh. Birman and T. A. Suslina, Threshold approximations with corrector for the resolvent of a factorized selfadjoint operator family, Algebra i Analiz 17 (2005), no. 5, 69-90; English transl., St. Petersburg Math. J. 17 (2006), no. 5, 745-762. MR2241423 (2008d:47047)

[156] - Homogenization with corrector term for periodic elliptic differential operators, Algebra i Analiz 17 (2005), no. 6, 1-104; English transl., St. Petersburg Math. J. 17 (2006), no. 6, 897-973. MR 2202045(2006k:35011)

[157] A. B. Alekseev, M. Sh. Birman, and N. D. Filonov, Spectrum asymptotics for one "nonsmooth" variational problem with solvable constraint, Algebra i Analiz 18 (2006), no. 5, 1-22; English transl., St. Petersburg Math. J. 18 (2007), no. 5, 681-697. MR2301038 (2008b:35193)

[158] M. Sh. Birman and T. A. Suslina, Homogenization with corrector for periodic differential operators. Approximation of solutions in the Sobolev class $H^{1}\left(\mathbb{R}^{d}\right)$, Algebra i Analiz 18 (2006), no. 6, 1-130; English transl., St. Petersburg Math. J. 18 (2007), no. 6, 857-955. MR2307356 (2008d:35008)

[159] _ Homogenization of a stationary periodic Maxwell system in the case of constant magnetic permeability, Funktsional. Anal. i Prilozhen. 41 (2007), no. 2, 3-23; English transl., Funct. Anal. Appl. 41 (2007), no. 2, 81-98. MR2345036 (2008i:35014)

[160] M. Sh. Birman and N. D. Filonov, Weyl asymptotics of the spectrum of the Maxwell operator with non-smooth coefficients in Lipschitz domains, Nonlinear Equations and Spectral Theory, Amer. Math. Soc. Transl. Ser. 2, vol. 220, Amer. Math. Soc., Providence, RI, 2007, pp. 27-44. MR2343605 (2009a:35243)

[161] M. Sh. Birman and T. A. Suslina, Operator error estimates in the homogenization problem for nonstationary periodic equations, Algebra i Analiz 20 (2008), no. 6, 30-107; English transl., St. Petersburg Math. J. 20 (2009), no. 6, 873-928. MR2530894 (2010f:35017)

[162] _ The limiting absorption principle and the homogenization procedure for periodic elliptic operators, Funktsional. Anal. i Prilozhen. 42 (2008), no. 4, 105-108; English transl., Funct. Anal. Appl. 42 (2008), no. 4, 336-339. MR2492431 (2010f:35016)

[163] M. Sh. Birman and T. A. Suslina, Homogenization of periodic differential operators as a spectral threshold effect, New Trends in Mathematical Physics. Selected Contributions of the XVth International Congress on Mathematical Physics, Springer, Berlin, 2009, pp. 667-683.

[164] M. Sh. Birman and V. A. Sloushch, Two-sided estimates for the trace of the difference of a pair of semigroups, Funktsional. Anal. i Prilozhen. 43 (2009), no. 3, 26-32; English transl., Funct. Anal. Appl. 43 (2009), no. 3, 184-189. MR2583637(2010k:47082)

[165] _ Discrete spectrum of the periodic Schrödinger operator with a variable metric perturbed by a nonnegative potential, Math. Model. Nat. Phenom. 5 (2010), no. 4, 32-53. MR2662449 (2011e:47085) 
[166] M. Sh. Birman and T. A. Suslina, The analog of the limiting absorption principle in homogenization of periodic elliptic operators, Operator Theory and its Applications: In Memory of V. B. Lidskii (1924-2008), Amer. Math. Soc. Transl. Ser. 2, vol. 231, Amer. Math. Soc., Providence, RI, 2010, pp. 35-57. MR2509773

\section{BoOKS}

[B1] M. Sh. Birman, N. Ya. Vilenkin, E. A. Gorin et al. Functional analysis. Ed. by S. G. KreĬn. Second edition, revised and augmented. Nauka, Moscow, 1972, 544 pp. (Russian) MR0352920 (50:5406)

[B2] M. Sh. Birman and M. Z. Solomyak. Spectral theory of selfadjoint operators in Hilbert space. Leningrad. Univ., Leningrad, 1980, 264 pp. (Russian) MR0609148 (82k:47001)

[B3] _ Quantitative analysis in Sobolev embedding theorems and applications to spectral theory, Transl. from Russian by F. A. Cezus, Amer. Math. Soc. Transl. Ser. 2, vol. 114, Amer. Math. Soc., Providence, RI, 1980. MR0562305 (80m:46026)

[B4] _ Spectral theory of selfadjoint operators in Hilbert space, Transl. from the 1980 Russian original by S. Khrushchev and V. Peller, Math. Appl. (Soviet Series), D. Reidel Publishing Co., Dordrecht, 1987. MR1192782 (93g:47001)

[B5] M. Sh. Birman. Selected works. Mathematical scattering theory. Spectral shift function. RKhD, Moscow-Izhevsk, 2010, 502 pp. (Russian)

[B6] M. Sh. Birman and M. Z. Solomyak. Spectral theory of selfadjoint operators in Hilbert operators. Second edition, revised and augmented. Lan', St. Petersburg, 2010, 464 pp. (Russian)

\section{Editorial Activity}

[E1] P. D. Laks and R. S. Fillips. Scattering theory. With a translation of "Decaying modes for the wave equation in the exterior of an obstacle" (Comm. Pure Appl. Math. 22 (1969), 737-787). Transl. from the English by N. K. Nikol'skiı̌ and B. S. Pavlov. Edited by M. Sh. Birman. Mir, Moscow, 1971, 312 pp. (Russian) MR0397448 (53:1307)

[E2] Problems of mathematical physics. No. 1. Spectral theory and wave processes. Edited by M. Sh. Birman. Leningrad. Univ., Leningrad, 1966, 133 pp. English transl., Topics in Mathematical Physics. Vol. 1. Consultants Bureau, Plenum Publishing Corporation, New York, 1967, viii+114 pp. MR0202524(34:2393), MR0210536 (35:1428)

[E3] Problems of mathematical physics. No. 2. Spectral theory. Diffraction problems. Edited by M. Sh. Birman. Leningrad. Univ., Leningrad, 1967, 158 pp. English transl., Topics in Mathematical Physics. Vol. 2. Consultants Bureau, New York, 1968, vii+134 pp. MR0233654(38:1975) MR0266727 (42:1630)

[E4] Problems of mathematical physics. No. 3. Spectral theory. Edited by M. Sh. Birman. Leningrad. Univ., Leningrad, 1968, 104 pp. English transl., Topics in Mathematical Physics. Vol. 3. Consultants Bureau, New York-London, 1969, vi+93 pp. MR0339968(49:4725) MR0345774(49:10505)

[E5] Problems of mathematical physics. No. 4. Spectral theory. Wave processes. Edited by M. Sh. Birman. Leningrad. Univ., Leningrad, 1970, 135 pp. English transl., Topics in Mathematical Physics. Vol. 4. Consultants Bureau, New York-London, 1971, v+121 pp. MR0267296 (42:2198) MR0339975 (49:4732)

[E6] Problems of mathematical physics. No. 5. Spectral theory. Edited by M. Sh. Birman. Leningrad. Univ., Leningrad, 1971, 128 pp. English transl., Topics in Mathematical Physics. Vol. 5. Consultants Bureau, New York-London, 1972. MR0300139 (45:9187. MR.0339976 (49:4733)

[E7] Problems of mathematical physics. No. 6. Theory of functions. Spectral theory. Wave propagation. Edited by M. Sh. Birman. Leningrad. Univ., Leningrad. 1973, 149 pp. (Russian) MR0338529 (49:3293)

[E8] Problems in mathematical physics. No. 7. Theory of functions. Spectral theory. Theory of wave propagation. Edited by M. Sh. Birman. Leningrad. Univ., Leningrad, 1974, 185 pp. (Russian) MR0424461 (54:12423)

[E9] Problems in mathematical physics. No. 8. Differential equations. Spectral theory. Theory of wave propagation. Edited by Birman. Leningrad. Univ., Leningrad 1976, 177 pp. (Russian) MR0424462 (54:12424)

[E10] Problems in mathematical physics. No. 9. Scattering theory. Theory of oscillations. Edited by M. Sh. Birman. Leningrad. Univ., Leningrad, 1979, 188 pp. (Russian) MR0566382 (81a:35003)

[E11] Problems in mathematical physics. No. 10. Spectral theory. Wave processes. Edited by M. Sh. Birman. Leningrad. Univ., Leningrad, 1982, 303 pp. (Russian) MR0691140 (84b:35004)

[E12] Problems in mathematical physics. No. 11. Differential equations. Scattering theory. Edited by M. Sh. Birman. Leningrad. Univ., Leningrad, 1986, 280 pp. (Russian) MR0857103 (87f:35002) 
[E13] Problems in mathematical physics. No. 12. Wave propagation. Scattering theory. Edited by M. Sh. Birman. Leningrad. Univ., Leningrad, 1987, 260 pp; English transl., Amer. Math. Soc. Transl. (2), vol. 157, Amer. Math. Soc., Providence, RI, 1993. MR0923966 (88j:00016) MR1252219 (94g:00020)

[E14] Problems in mathematical physics. No. 13. Differential equations. Spectral theory. Wave propagation. Edited by M. Sh. Birman. Leningrad. Univ., Leningrad, 1991, 311 pp. (Russian) MR1341627 (96b:00011)

[E15] Estimates and asymptotics for discrete spectra of integral and differential equations. Papers from the Seminar on Mathematical Physics held in Leningrad, 1989-90. Edited by M. Birman. Transl. from Russian. Adv. in Soviet Math., vol. 7. Amer. Math. Soc., Providence, RI, 1991, 204 pp. MR.1141209 (92g:35153)

[E16] Nonlinear problems in mathematical physics and related topics. I. In honor of Academician O. A. Ladyzhenskaya. Edited by M. Sh. Birman, S. Hildebrandt, V. A. Solonnikov, and N. N. Ural'tseva. Mezhdunar. Mat. Ser., vol. 1, Tamara Rozhkovskaya, Novosibirsk, 2002; English transl., Kluwer/Plenum Publishers, New York, 2002. MR1971549 (2003m:35003)

[E17] Nonlinear problems in mathematical physics and related topics. II. In honor of Academician O. A. Ladyzhenskaya. Edited by M. Sh. Birman, S. Hildebrandt, V. A. Solonnikov, and N. N. Ural'tseva, Mezhdunar. Mat. Ser., vol. 2, Tamara Rozhkovskaya, Novosibirsk, 2002; English transl., Kluwer/Plenum Publishers, New York, 2002. MR1971985 (2003m:35004)

[E18] Nonlinear equations and spectral theory. Edited by M. Sh. Birman and N. N. Uraltseva. Amer. Math. Soc. Transl. Ser. 2, vol. 220. Adv. in Math. Sci., vol. 59. Amer. Math. Soc., Providence, RI, 2007, 246 pp. MR2343603 (2008c:35002)

\section{List of STUdents of M. Sh. Birman}

1. *Pavlov Boris Sergeevich (Ph.D. - 1963)

2. Entina Sofia Borisovna (M.Sc. - 1960)

3. Skvortsov Genrikh Evgen'evich (M.Sc. - 1961)

4. Verzhbinskii Gleb Mikhailovich (Ph.D. - 1968)

5. *Zheludev Valerii Aleksandrovich (Ph.D. - 1968)

6. Kurenbin Oleg Ivanovich (M.Sc. - 1966)

7. Deich Vladimir Genrihovich (Ph.D. - 1971)

8. ${ }^{*}$ Borzov Vadim Vasil'evich (Ph.D. - 1971)

9. Rotfeld Sergey Yul'evich (Ph.D. - 1972)

10. Koplienko Leonid Sergeevich (Ph.D. - 1973)

11. *Yafaev Dmitrii Rauel'evich (Ph.D. - 1973)

12. *Vasyunin Vasilii Ivanovich (M.Sc. - 1972)

13. Belopol'skii Andrey L'vovich (Ph.D. - 1975)

14. *Naidhardt Hagen (M.Sc. - 1975)

15. Alekseev Aleksandr Borisovich (Ph.D. - 1977)

16. Suslov Aleksandr Vital'evich (M.Sc. - 1978)

17. ${ }^{*}$ Korotyaev Evgenii Leonidovich (Ph.D. - 1982)

18. Firsova Nataliya Evgen'evna (Ph.D. - 1983)

19. Sayapova Margarita Robertovna (M.Sc. - 1980)

20. Andreev Aleksei Sergeevich (Ph.D. - 1984)

21. Solnyshkin Sergey Nikolaevich (Ph.D. - 1984)

22. Sobolev Aleksandr Vladimirovich (M.Sc. - 1982)

23. *Raikov Georgii Dimitrov (Ph.D. - 1986)

24. *Suslina Tatiana Aleksandrovna (Ph.D. - 1987)

25. Khryashchev Sergey Viktorovich (Ph.D. - 1994)

26. *Weidl Timo (M.Sc. — 1994)

27. Safronov Oleg Leonidovich (Ph.D. - 1997)

28. Pushnitskii Aleksandr Borisovich (Ph.D. - 1998)

29. Filonov Nikolai Dmitrievich (Ph.D. - 1999)

30. Sloushch Vladimir Anatol'evich (Ph.D. - 2000) 
31. Shterenberg Roman Grigor'evich (Ph.D. - 2003)

32. Frank Rupert (M.Sc. - 2003)

33. Morozov Sergey Vladimirovich (M.Sc. - 2005)

The years of the Ph.D. thesis defence or M.Sc. thesis defence under M. Sh. Birman supervision are given in parenthesis. For those students who defended Ph.D. thesis under supervision of M. Sh. Birman, we omit the date of M.Sc. thesis defence. The information concerning Ph.D. students is complete. As for M.Sc. students, only information known to the authors (possibly, not complete) is given. Those who later became Doctors of Sciences are marked by *

Department of Mathematics, The Weizmann Institute of Science, Rehovot 76100, Israel

E-mail address: michail.solomyak@weizmann.ac.il

St. Petersburg State University, Department of Physics, Ul'yanovskaya 3, Petrodvorets, St. Petersburg 198504, Russia

E-mail address: suslina@list.ru

IRMAR, University Rennes-1, Rennes 35042, France

E-mail address: dimitri.yafaev@univ-rennes1.fr

Received 31/OCT/2010

Translated by T. A. SUSLINA 